\title{
Metal-Organic Frameworks and Their Composites Towards Biomedical Applications
}

\author{
Yana Ma ${ }^{1,2}$, Xianglong $Q u^{3}$, Cui Liu ${ }^{1,2}$, Qiuran $X u^{4,5 * t}$ and Kangsheng $T u^{3 * t}$ \\ ${ }^{1}$ School of Basic Medical Sciences, Xi'an Key Laboratory of Immune Related Diseases, Xi'an Jiaotong University, Xi'an, China, \\ ${ }^{2}$ Key Laboratory of Environment and Genes Related to Diseases, Xi'an Jiaotong University, Ministry of Education, Xi'an, China, \\ ${ }^{3}$ Department of Hepatobiliary Surgery, the First Affiliated Hospital of Xi'an Jiaotong University, Xi'an, China, ${ }^{4}$ Laboratory of Tumor \\ Molecular Diagnosis and Individualized Medicine of Zhejiang Province, Zhejiang Provincial People's Hospital, Affiliated People's \\ Hospital, Hangzhou Medical College, Hangzhou, China, ${ }^{5}$ Research Center of Diagnosis and Treatment Technology for \\ Hepatocellular Carcinoma of Zhejiang Province, Hangzhou, China
}

Edited by:

Chunhua Yang,

Georgia State University,

United States

Reviewed by:

Dingpei Long,

Georgia State University,

United States

Yong-Qiang Li,

Shandong University, China

Cheng $\mathrm{Li}$,

Beihang University, China

Yang Xuan,

Dalian Minzu University, China

${ }^{*}$ Correspondence:

Qiuran Xu

windway626@sina.com

Kangsheng Tu

tks0912@foxmail.com

TORCID:

Qiuran Xu

orcid.org/0000-0003-2171-9279

Kangsheng Tu

orcid.org/0000-0002-0032-1459

Specialty section:

This article was submitted to

Nanobiotechnology,

a section of the journal

Frontiers in Molecular Biosciences

Received: 30 October 2021 Accepted: 22 November 2021

Published: 21 December 2021

Citation:

Ma Y, Qu X, LiU C, Xu Q and TU K (2021) Metal-Organic Frameworks and

Their Composites Towards

Biomedical Applications.

Front. Mol. Biosci. 8:805228.

doi: 10.3389/fmolb.2021.805228
Owing to their unique features, including high cargo loading, biodegradability, and tailorability, metal-organic frameworks (MOFs) and their composites have attracted increasing attention in various fields. In this review, application strategies of MOFs and their composites in nanomedicine with emphasis on their functions are presented, from drug delivery, therapeutic agents for different diseases, and imaging contrast agents to sensor nanoreactors. Applications of MOF derivatives in nanomedicine are also introduced. Besides, we summarize different functionalities related to MOFs, which include targeting strategy, biomimetic modification, responsive moieties, and other functional decorations. Finally, challenges and prospects are highlighted about MOFs in future applications.

Keywords: metal-organic frameworks, nanomedicine, therapeutics, imaging, theranostics, sensors

\section{INTRODUCTION}

Nanomedicine, aiming to solve health and medicine problems using nanomaterials with size at nanometer scales, has become a promising field. Applications of nanomedicine include developing delivery systems, contrast agents, sensors for effective chemotherapy, and diagnostics (Nance, 2019). Nanomaterials play an irreplaceable role in nanomedicine, with various resultant materials, such as organic materials, inorganic nanoparticles, and organic-inorganic hybrids, of which metal-organic frameworks (MOFs) are promising platforms in disease theranostics.

MOFs, also named porous coordination polymer (PCP), are constructed by metal ions or clusters covalently binding to organic linkers, which have attained increasing attention of investigations in various fields, from industry such as energy to medicine for therapeutics. As fascinating materials, MOFs exhibit the following exciting superiorities compared with other current existing nanomaterials: 1) versatile MOFs could be easily obtained through kinds of metal ions and organic linkers. Metal ions commonly applicable include zinc $(\mathrm{Zn})$, iron $(\mathrm{Fe})$, cobalt $(\mathrm{Co})$, and zirconium (Zr), while organic linkers include imidazolates, carboxylates, and phenolates. In addition, as various metals and linkers are available, nontoxic or lower toxic precursors could be chosen for high biocompatible MOFs. 2) High specific surface area and tailorable porosity endow MOFs with high loading efficiency, while the tunable size makes it possible for MOFs to be transported throughout the body through enhanced permeability and retention (EPR) effect. 3) As coordination bonds between precursors are labile, MOFs could be degraded to small molecules and excreted out 


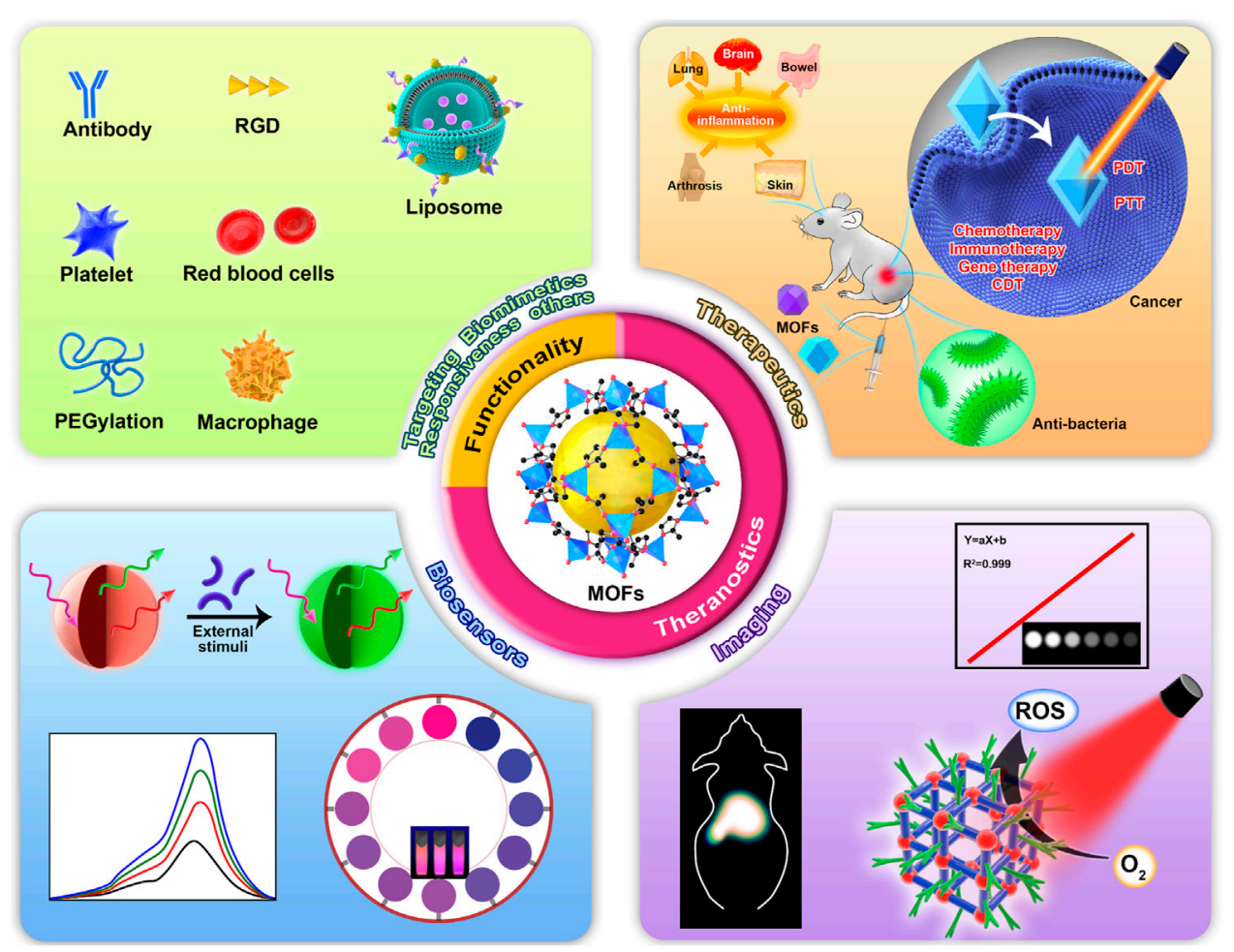

FIGURE 1 | Schematic illustration of functionalities and biomedical applications of metal-organic frameworks (MOFs).

from the body. 4) MOFs are modifiable through surface chemistry and defect structure, which could extend the function of MOFs such as multi-responsiveness and precise targeting (Wang et al., 2018b). All of these features make MOFs ideal candidates for nanomedicine, such as drug delivery, imaging, and sensor. Moreover, MOF composites fabricated by integrating MOFs with functional entities such as nanoparticles and polymer not only retain the structures and functions of MOFs but also endow additional functions to MOFs, which are more favorable for clinical applications ( $\mathrm{Zhu}$ and $\mathrm{Xu}$, 2014; Li and Huo, 2015).

This review aims to summarize the applications of MOFs and their composites in nanomedicine in the last 5 years (Figure 1). Different from various excellent reviews about MOFs, this review mainly emphasizes the strategies/function-orientated therapeutics, imaging, sensors, and theranostics of MOFs and their composites. Also, we outline the kinds and functions of the current surface functionalities of MOFs. In addition, we mention the applications of MOF derivatives. Finally, challenges and perspectives of MOFs and their hybrids in future applications are presented.

\section{FUNCTIONALIZATION}

Functionalization is achieved by adding new functions, features, capabilities, or properties to materials by changing their surface chemistry. Apart from high cargo loading, materials must meet numerous other requirements for precise function in vivo. With high biocompatibility, longer circulation time, and the ability to evade the immune system, MOFs can be flushed out from the bloodstream and arrive at the lesion sites through the passive EPR effect. However, drugs that accumulate in lesion sites only through the EPR effect are limited; thus, more effective strategies to improve drug accumulation are needed. By grafting functional targeting entities to the surface of MOFs, they could actively and efficiently get to target sites. In addition, to avoid premature release, it is vital to modify moieties such as PEG to the surface of MOFs. Therefore, this chapter is emphasized on the kinds and applications of surface functionalization of MOFs (Figure 2).

\subsection{Targeting Modification}

Nanomaterials play their roles only when they reach the lesion sites through either passive targeting or active targeting. Conventionally, nanoparticles rely on the passive EPR effect to arrive at the target sites. However, drug accumulation by this way often has unsatisfactory therapeutic effects, with active targeting needed. Active targeting could be mediated by ligand, aptamer, antibody, etc., with all of them depending on the interaction between targeting moieties and specific receptors of target sites.

\subsubsection{Hyaluronic Acid-Mediated Targeting}

Among various targeting ligands, hyaluronic acid (HA) is a widely used ligand for tumor targeting. HA, a negative linear polysaccharide that exists in the extracellular matrix (ECM), 


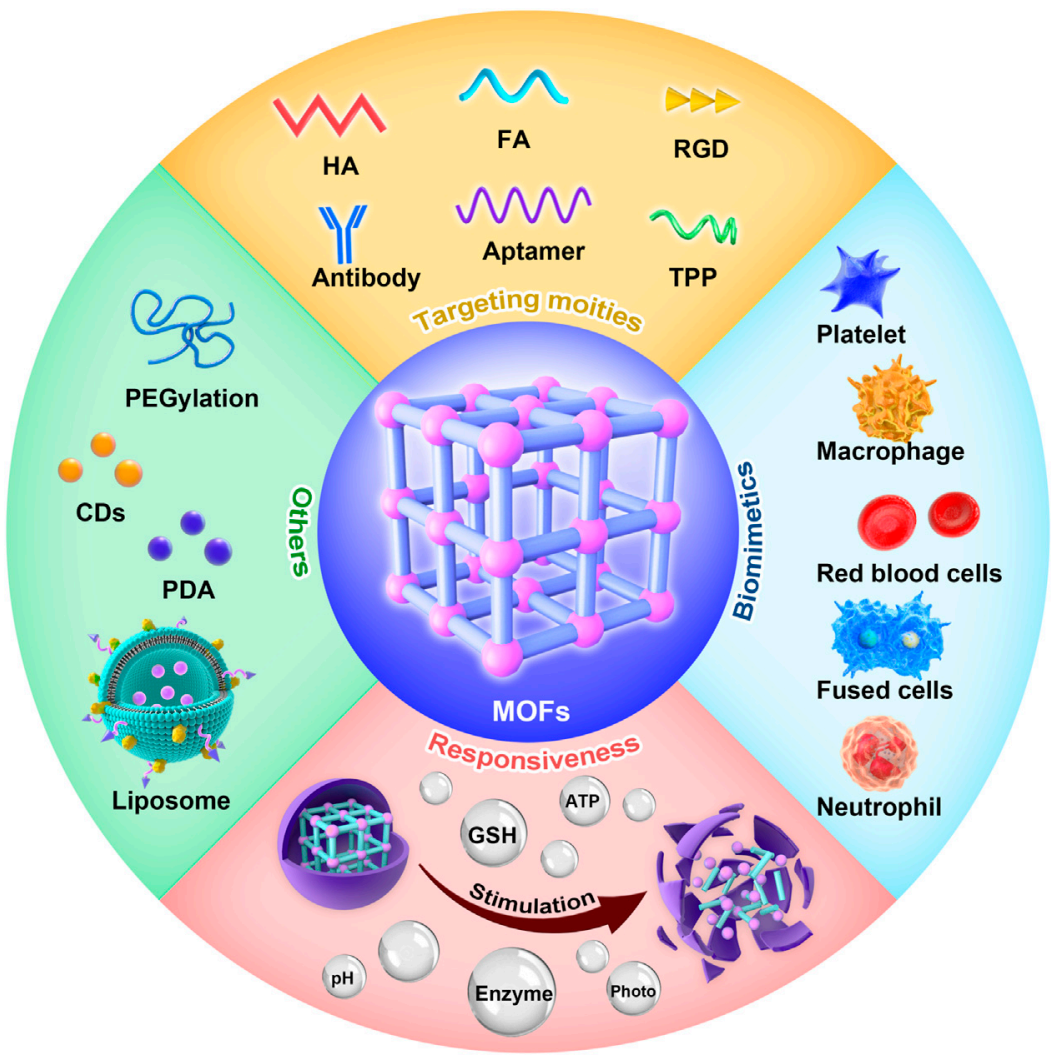

FIGURE 2 | Functionalities of metal-organic frameworks (MOFs), including targeting moieties, biomimetics, and responsiveness.

could be recognized by cluster determinant 44 (CD44) overexpressed on the surface of most cancer cells. With excellent biocompatibility and high biodegradability, it has promising applications in nanomedicine (Swierczewska et al., 2016). Inspired by this, various HA-coated MOFs were established for effective targeting through the interaction between HA and MOFs. For example, Sun et al. fabricated a HA/a-TOS@ZIF-8 nanosystem, in which the shell of HA was both an intelligent "switch" and a tumor guider for nanoparticle accumulation in tumor sites via CD44-mediated pathway. Compared with nanoparticles without HA coating, stronger fluorescence (FL) intensity in cells was observed after treatment with HA/ -TOS@ZIF-8 NPs, indicating that HA coating increased the internalization of NPs by cancer cells. Notably, in the tumor microenvironment, HA could be disintegrated by hyaluronidase (HAase), and thus, the MOFs were exposed, and the drugs were further released (Sun et al., 2019). Apart from tumor regions, HA coating could target inflammation sites and also improve the water stability of MOFs. Significantly, $\mathrm{HA}$ on the surface of MOFs also alleviates inflammation by reducing the expression of cytokines (Xiong et al., 2020).

\subsubsection{Folic Acid-Mediated Targeting}

Many cancer cells, including breast, cervical, colorectal, ovarian cancer cells, express a high level of folate receptors (FRs) on their cell surface, and folic acid (FA) is a universally studied targeting agent. As moiety provides ease of modification, FA could bind to various nano-MOFs through interaction between carboxylic groups of $\mathrm{FA}$ and positive moieties, such as $\mathrm{Hf}_{6}$ cluster of HfMn-NMOF (Bao et al., 2020), amino groups of Uio-66- $\mathrm{NH}_{2}$, and IRMOF-3 (Yang et al., 2017a; Gao et al., 2018b). Besides, FA could also be grafted to MOFs through intermediates, exemplified by binding FA to PEI on the surface of gadolinium-porphyrin MOFs (FA-NPMOFs) (Chen et al., 2019b).

\subsubsection{RGD Peptide-Mediated Targeting}

Integrin family, as adhesion molecules, is involved in mediating the interaction of cell-cell and cell-ECM and regulates the majority of cell processes including cell proliferation and migration, which were found to be pathophysiologically activated in many cell types of diseases. Arg-Gly-Asp (RGD) tripeptide motif, highly expressed in some tumor cells, has a high affinity to adhesion molecules and integrin $\alpha_{V} \beta_{3}$ (Dong et al., 2017b) and thus plays essential roles in drug delivery for cancer treatment. Studies have demonstrated that RGD-modified ZIF-8 could target and treat cancer cells (Dong et al., 2019). Other RGD-containing peptides, such as iRGD (amino acid sequence: CRGDK/RGPD/EC), could also target cancer cells through binding to neuropilin-1 (NRP-1) receptors expressed in tumor and vascular tissues (Liu et al., 2021b). 


\subsubsection{Aptamer-Mediated Targeting}

Aptamers are single-stranded DNA or RNA molecules with high specificity and affinity to their targets. AS1411, a 26-mer DNA aptamer with guanine-rich DNA segment known as G-quadruplex structure, could bind to nucleolin highly expressed both in the cell and on the cell surface (YazdianRobati et al., 2020) and thus has attracted increasing interest in targeting treatment. AS1411 has been successfully introduced to various MOFs or their composites, such as ZIF-8 (Dong et al., 2018), $\gamma$-CD-MOF (Jia et al., 2019), and FeTCPP/ $\mathrm{Fe}_{2} \mathrm{O}_{3} \mathrm{MOF}$ (Zhao et al., 2020b). Furthermore, to further enhance the targeting efficiency, in an experiment for targeting VEGFoverexpressed cancer cells, Chen et al. constructed a doxorubicin (DOX)-loaded $\mathrm{Zr}^{4+}$-MOF with dual aptamer, VEGF aptamer, and AS1411 aptamer. On the one hand, this nanoparticle could target cancer cells through AS1411 aptamer; on the other hand, in the presence of VEGF, the MOF could be unlocked and the DOX was released, thus accurately killing the tumor (Chen et al., 2018d).

\subsubsection{Other Targeting Functionalities}

The antibody is a universal and powerful tool for creating specific cell targeting through antigen-antibody interaction. Cherkasov et al. engineered an antibody-directed magnetic core-shell MOF through covalently binding anti-HER2 antibody to functional polymer on the surface of MOF, which could target and kill HER2/neu-positive cancer cells (Cherkasov et al., 2020). In addition, primary amine-containing MOF could also conjugate to an antibody, which could be used for antibody-targeting strategy. For example, Eu-2-amino-BDC material with an available primary amine in its organic linker could bind to EpCAM antibody, which guided MOFs to human epithelial cell A549 for imaging (Butler et al., 2020).

Mitochondria, one of the essential organelles for various cell functions and energy production, is a promising therapeutic target (Cho et al., 2020). Of all target moieties (GuzmanVillanueva and Weissig, 2017), triphenylphosphine (TPP) is most commonly applicable in MOF nanomedicine from simple Zr-MOF (Zhou et al., 2018a) to composites synthesized by hybridization of porphyrin MOF with UCNPs (Liu et al., 2020).

The molecular marker is another potentially promising targeting approach. For example, hyperphosphorylated tau, as the main feature of Alzheimer's disease (AD), could be used as a potential target. Immobilized targeting reagent 5-amino-3(pyrrolo[2,3-c]pyridin-1-yl)isoquinoline (defluorinated MK6240, DMK6240) to the surface of MOF Fe-MIL-88B$\mathrm{NH}_{2}$-NOTA-DMK6240/MB could be precisely located in the lesion sites by targeting hyperphosphorylated tau specifically, which then ameliorated $\mathrm{AD}$ symptom through inhibiting the aggregation status of hyperphorylated tau (Zhao et al., 2020a).

\subsection{Biomimetic Modification}

Various cell membranes, with their superiority, such as long circulation time and homotypic targeting, have gained increasing attention in bioinspired camouflage strategy. Membrane coatings on MOFs endow them with the superiority of cell membrane.
And applicable cell membranes include erythrocyte membrane, platelet membrane, white blood cell membrane, cancer cell membrane, and hybrid cell membrane.

\subsubsection{Erythrocyte Membrane/Red Blood Cell Membrane}

Red blood cell (RBC) membranes are the first and widely used cell membranes in nanomedicine, as they have a long life span of about 120 days. In addition, with self-marker CD47 and a series of complement regulatory proteins, RBCs exhibit a strong ability of immune evasion. Inspired by these specialties, Zhang et al. fabricated TGZ@eM nanoreactor with erythrocyte membrane coated on ZIF-8 for co-delivery of glucose oxidase (GOx) and prodrug tirapazamine (TPZ). In vivo pharmacokinetics results showed the half-time of TGZ@eM $\left(\mathrm{t}_{1 / 2}=4.7\right)$ was two times longer than that of $\operatorname{TGZ}\left(t_{1 / 2}=2.4\right)$, which enhanced the accumulation of TGZ@eM to the tumor. In addition, in vitro cell uptake experiments demonstrated that only a few Rhm B-GOx-ZIF modified with erythrocyte membrane could be taken up by RAW264.7 cells, indicating a better immune escape ability. Owing to these properties of more prolonged blood circulation, better immune evasion, and better tumor targeting, this TCZ@eM exerted better performance for killing tumors (Zhang et al., 2018b) (Figure 3). Similar effects were conducted in $\mathrm{O}_{2}$ and ICG-loaded RBC-UiO-66 NPs, wherein RBC membrane-derived $\mathrm{O}_{2}$ @UiO-66@ ICG@RBC nanoparticles accumulated in tumor site through their longer circulation ability and immunity-evading superiority and then enhanced the effect of photodynamic therapy (PDT) (Gao et al., 2018a). However, single RBC membrane coating still showed not enough targeting ability; thus, various targeting ligands were integrated into the cell membrane to improve targeting specialty. For example, by modifying erythrocyte membrane with tumor-targeting peptide cyclic RGD (cRGD) and then decorating them onto DOX-loaded ZIF-8, the resultant DOX @ZIF-8@eM-cRGD exhibited both longer blood retention time and specific tumor targeting, which thus improved the tumor inhibition (Lin et al., 2020).

\subsubsection{Platelet Membrane}

Platelets, fragments released from megakaryocytes, play critical roles in hemostasis. After trauma, platelets could quickly adhere to the injured vascular and trigger the process of coagulation. Platelet membranes exhibit similar circulation properties with RBC; they could also actively target the tumor and inflammation sites through CD44 receptors. Therefore, MOFs coated with platelet membrane might not only possess immunomodulatory ability and capability of selective adhesion to lesion sites but also avoid macrophage uptake (Hu et al., 2015; Fang et al., 2018). This was demonstrated by Zhuang et al. through cloaking platelet membrane onto siRNA-encapsulated ZIF-8. In vitro cell uptake experiment showed that platelet membrane decorating endowed nanoparticles with immune evasion ability with less uptake by macrophage. In addition, the targeting ability of SK-BR-3 cells was also improved. Accordingly, the tumor localization was demonstrated in both in vivo and ex vivo experiments (Zhuang et al., 2020). 

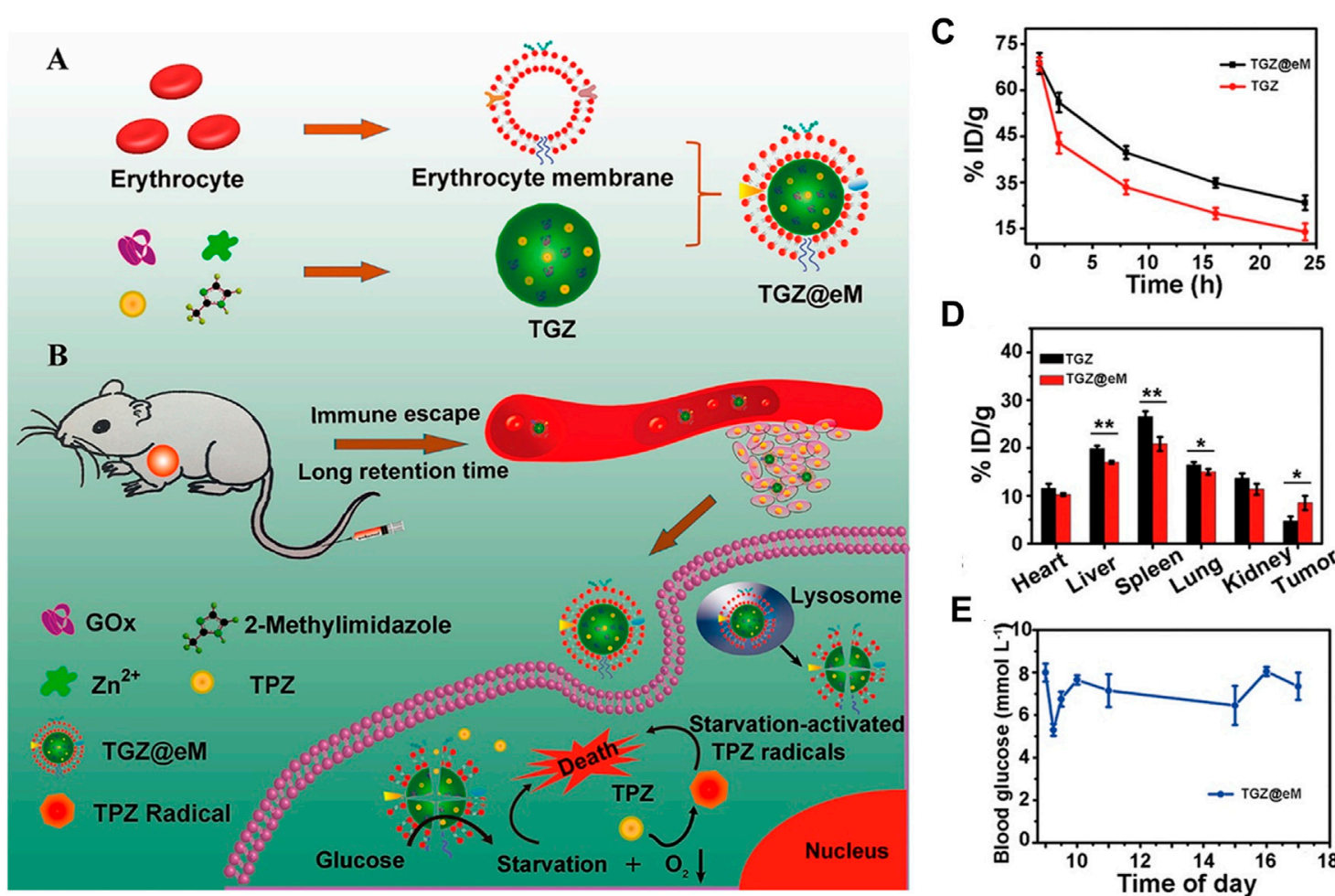

D

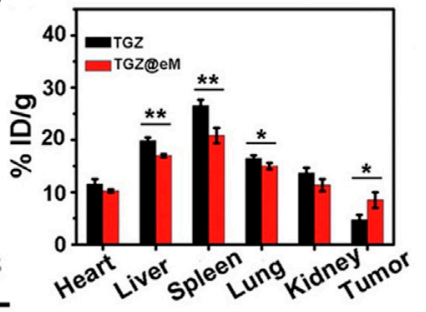

$\mathbf{E}$

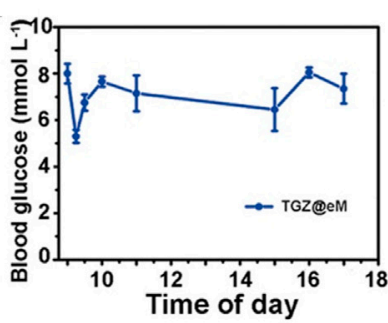

FIGURE 3 | Synthesis, application, and pharmacokinetics of erythrocyte membrane-cloaked metal-organic frameworks (MOF), TGZ@eM. (A) Schematic illustration of TGZ@eM synthesis. (B) Application diagram of TGZ@eM in cancer treatment. (C) In vivo pharmacokinetics of TGZ@eM and TGZ. (D) Biodistribution of TGZ@eM and TGZ. (E) Blood glucose level of mice during daytime after injection with TGZ@eM. Reproduced with permission. Copyright 2018, ACS Nano.

\subsubsection{White Blood Cell Membrane}

White blood cells consist of neutrophils, macrophages, dendritic cells (DCs), and so on. As a crucial component for the body's defense system, white cell membranes possess various membrane receptors and specific targeting moieties for targeting lesion sites including inflammation and tumor sites.

Macrophages, as innate immune cells, apart from the extended circulation property, could target lesion sites such as tumors, inflammation, and injured vasculatures. Based on these, Chen et al. designed a persistent luminescence nanoparticle@MOFderived mesoporous carbon core-shell nanocomposite coating by macrophage membrane (MPLMC). The cloaking ability of macrophages was evaluated, and results showed that MPLMC exhibited weaker luminescence than PLMC and PLMC-PEG without membrane coating. In addition, both in vivo and ex vivo experiments demonstrated the stronger targeting ability for tumors after membrane cloaking (Chen et al., 2020).

Neutrophils, the first white blood cells recruited into lesion sites after trauma, have the natural ability to target inflammation/ tumor sites through the transmigration process. By the inflammation-targeting ability of neutrophils, Zhang's group designed a neutrophil membrane biomimetic nanoplatform. In this nanoplatform, neutrophil membranes were cloaked on a porphyrinic porous coordination network (PCN) decorated by silver nanoparticles (AgNPs). In vivo imaging results demonstrated that this nanoparticle was increasingly accumulated at the tumor site with maximum accumulation at $24 \mathrm{~h}$ after intravenous administration (Zhang et al., 2020a).

\subsubsection{Cancer Cell Membrane}

Cancer cell membranes can escape the supervision of the immune system and bind to tumor cells through homotypic adhesion, which made them well-suited and widely used in tumor-targeting nanomedicine. Li et al. developed a cancer cell membrane cloaked cascade bioreactor (mCGP) for synergistic treatment of cancer. In this bioreactor, $\mathrm{GOx}$ and catalase (CAT) were embedded into PCN-224, followed by cancer cell membrane coating. After incubation with nanoparticles, 4T1 cells exhibited 2.8-fold stronger FL intensity than African green monkey kidney (COS7) normal cells and other cancer cells. Similar results were conducted in tumor accumulation in vivo. Moreover, 2.5-fold weaker FL was observed after treating RAW264.7 cells with mCGP than that of PCN-224. All these results demonstrated that cancer cell membrane coating not only endowed nanoparticles with immune evasion ability but also enhanced the tumor-targeting ability (Li et al., 2017). An et al. combined this superiority of cancer cell membrane with dual-responsive multimodal nanoformulation to realize the gassonodynamic combined treatment (An et al., 2020b). More interestingly, incubating different cancer cell membranes with MOF-loaded CRISPR/Cas9 could deliver the CRISPR/Cas9 system to specific cells to realize the personalized gene 


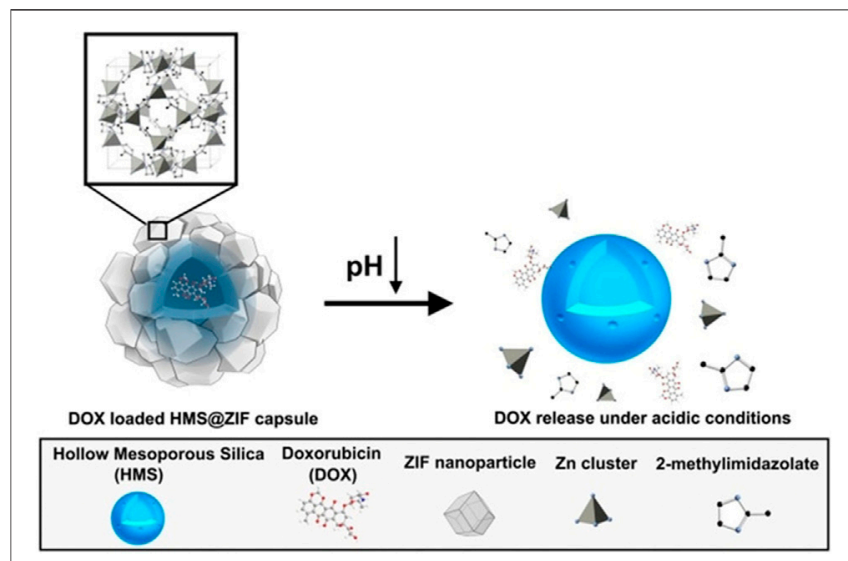

FIGURE 4 | Schematic illustration of hollow mesoporous silica/ metal-organic framework (HMS/MOF) pH responsiveness. Reproduced with permission. Copyright 2018, ChemMedChem.

knockout (Alyami et al., 2020), which is a promising direction for disease treatment.

\subsubsection{Hybrid Cell Membrane}

Although cell membrane biomimetics owns a tremendous advantage for nanomedicine, single-cell membrane coating could not reach optimal strength owing to their limitations, which could be offset by hybrid cell membranes. Hybrid cell membranes, fused by two or more different cell lines, inherit the properties of dual or multiple cells and thus extend their functions (Liu et al., 2019d). By combination fusion cell membrane acquired from cancer cells and DCs with photosensitizer (PS)-containing MOFs, Zhang's group engineered an expandable immunotherapeutic nanoplatform (PCN@FM). This PCN@FM nanoparticle could target tumor sites both in vitro and in vivo. In addition, owing to the expression of cancer antigens and immunological co-stimulatory molecules, PCN@FM not only inhibited rebound of the primary tumor but also restrained distant tumors (Liu et al., 2019d).

\subsection{Stimulus Responsiveness}

Apart from biocompatibility and specific targeting, the controlled release of drugs is significant for optimal therapeutics. Therefore, establishing responsive nanomaterials to realize on-demand release is imperative. With advantages of diversity, flexible structure, and ease of modification, MOFs are such well-suited materials that made drug release possible at desired sites, which could respond to not only disease microenvironment, such as lower $\mathrm{pH}$ and higher glutathione (GSH), but also external conditions such as magnetic fields.

\subsection{1 pH Responsiveness}

$\mathrm{pH}$-responsive MOFs are widely investigated in nanomedicine due to the acidic conditions of lysosomes, tumors, and inflammation. MOFs with intrinsic acidic responsiveness include $\mathrm{Zn}-\mathrm{MOF}$, Fe-MOF, and $\mathrm{Zr}-\mathrm{MOF}$, which have been widely used in nanomedicine. ZIF-8 in the Zn-MOF family assembled by $\mathrm{Zn}^{2+}$ and 2-methylimidazole showed excellent $\mathrm{pH}$ sensitivity, which could be disrupted at low $\mathrm{pH}$ (5.0-6.0) (Dong et al., 2018; Liang et al., 2018; Dong et al., 2019). For instance, in the drug-release experiment of camptothecin@ZIF$8 @$ RGD, there was almost no drug release under $\mathrm{pH} 7.4$, while about $75 \%$ of drugs were released after $24 \mathrm{~h}$ in pH 5.0 (Dong et al., 2019). Moreover, composites fusing ZIF- 8 with other functional moieties such as polyacrylic acid (PAA) (Yan et al., 2017; Chen et al., 2019a) and hollow mesoporous silica (HMS) (Jia et al., 2018) would not change the $\mathrm{pH}$ sensitivity of ZIF-8. Taking DOX-loaded HMS@ZIF-8 hybrid materials as an example, less than $3 \%, 46.6 \%, 71.4 \%$, and $85 \%$ of DOX were released within $10 \mathrm{~h}$ in $\mathrm{pH} 7.4,6.0,5.0$, and 4.0, respectively (Jia et al., 2018) (Figure 4). In addition, by incorporating PAA into MOFs, higher $\mathrm{pH}$ responsiveness and better drug loading were achieved. ZIF-90 assembly of $\mathrm{Zn}^{2+}$ and imidazole-2-carboxaldehyde (IcaH) (Guan et al., 2018), IRMOF-3 composed of $\mathrm{Zn}^{2+}$ ions and 2aminoterephthalic acid (Liu et al., 2019c) were also pH driven. In addition, Fe-MOFs also exhibit $\mathrm{pH}$ sensitivity, and discharge of drug in drug-loaded MIL-100 and MIL-101(Fe) (Cabrera-Garcia et al., 2019; Gupta et al., 2019) was observed at pH 5, which boosted the intracellular release of drugs. Zr-porphyrin MOF(PCN-222) also showed $\mathrm{pH}$-responsive features with a cumulative release rate of $86.29 \%$ at $\mathrm{pH} 5.5$ compared with 63.23\% at pH 7.2 (Leng et al., 2018).

To those with no inherent $\mathrm{pH}$ sensitivity, immobilized functional groups could also realize the $\mathrm{pH}$ responsiveness. Chitosan (CS) on the $\mathrm{Fe}_{3} \mathrm{O}_{4} @ O C M C @$ IRMOF-3/FA (Chowdhuri et al., 2016), CS/Bio-MOF (Abazari et al., 2018), and hydroxyapatite (HAp) on the $\mathrm{Fe}_{3} \mathrm{O}_{4} @ \mathrm{Fe}-\mathrm{MOF} @ H a p$ (Yang et al., 2017b) were applicable for drugs on-demand release at lower $\mathrm{pH}$.

\subsubsection{Redox Responsiveness}

Redox environment such as a high level of GSH, especially in the tumor, is preferable for controlled release through constructing redox-responsive carriers. MOFs, by assembly with disulfide bond-containing ligands, or dissolution through redox reaction upon redox agents, or easy modification with sensitive molecules, are such materials that meet the requirements. For example, in vitro released results showed that cargoes in MOF-M (DTBA) $(\mathrm{M}=\mathrm{Fe}, \mathrm{Al}$, or $\mathrm{Zr})$ consisted of metal ions and $4,4^{\prime}$ dithiobisbenzoic acid (4,4'-DTBA) displayed faster release in the condition of GSH, which could be attributed the disulfide bond in $4,4^{\prime}$-DTBA being cleavable (Lei et al., 2018). In addition, a new type of drug delivery system based on thiol-functionalized Uio-66- $(\mathrm{SH})_{2}$ was developed for redox sensitiveness. In this system, benzoic acid (BA), as a modulator, competed with ligand 2,5-disulfanylterephthalic acid (BDC- $\left.(\mathrm{SH})_{2}\right)$ to $\mathrm{Zr6}$ clusters, thus forming small and large pores of Uio-66-( $\mathrm{SH})_{2}$ after removal of BA. Then thiol-containing anticancer drug 6mercaptopurine (6-MP) was loaded through a disulfide bond formed between thiol groups on BDC- $(\mathrm{SH})_{2}$ and 6-MP. Upon high concentration of GSH, the release of 6-MP was shown (Gong et al., 2020). In another study, Wang et al. selected Cu(II) carboxylate-based MOF-199 to carry PSs. Once internalized by cells, $\mathrm{Cu}(\mathrm{II})$ reacted with and depleted endogenous $\mathrm{GSH}$, induced 
decomposition of MOF-199, and released the PSs to ablate the tumor cells specifically (Wang et al., 2019d).

\subsubsection{Other Responsive Systems}

Apart from widely used $\mathrm{pH}$ and redox conditions, other stimuli such as ATP, light, and temperature are also applied for controlled release. Adenosine triphosphate (ATP) is the energy source of all biological processes, whose dysfunction is related to many diseases, especially cancer. Therefore, developing ATPresponsive nanosystems depending on the interaction between ATP and counterpart is beneficial for intelligent drug release. ZIF-90, upon ATP, would be degraded due to competitive binding between ATP and $\mathrm{Zn}^{2+}$, thus releasing the cargo (Yang et al., 2019). Another strategy such as disrupting the coordination between ATP and anti-ATP aptamer is also available. In a DOX-loaded MOF coated with nucleic acidbased polyacrylamide hydrogel, anti-ATP aptamer on the MOFs could bind to ATP overexpressed in cancer sites and thus realize the controlled release of DOX (Chen et al., 2018b). Light-responsive MOFs synthesized by assembly metal Zr with photo-responsive linker azobenzenedicarboxylate (AZB) were stable in the dark, while they were rapidly degraded upon irradiation with light at $340 \mathrm{~nm}$ (Roth Stefaniak et al., 2018). Also, a plasmonic core-shell gold nanostars/ZIF-8 was lightresponsive. Under near-IR (NIR) light irradiation, core gold nanostars created local temperature gradients and induced drug thermodiffusion (Carrillo-Carrion et al., 2019). Furthermore, redox-responsive selenium (Se)-substituted polymer coated onto porphyrin Zr-MOF (PCN-224) (Luo et al., 2019) also realized photo-induced release of the payload. Given the differential expression of enzymes at disease sites such as azoreductase, Fe-MOF composed of $\mathrm{Fe}^{3+}$ and $4,4^{\prime}-$ azobisbenzoic acid was introduced for enzyme-responsive MOF. This MOF could be decomposed by reducing the azobenzene units to an amine by overexpressed azoreductase in an oxygen-deficient environment, thus releasing the payloads in cancer lesion sites (Liu et al., 2019a; Huang et al., 2019).

\subsubsection{Multi-Responsive Nanosystems}

A single-responsive nanoplatform does not usually meet the precise release of payloads. Ren et al. prepared a $\mathrm{pH} /$ redox dualresponsive nanocarrier with an organosilica shell coated on ZIF8 . In an in vitro experiment, the release of DOX was only about $18 \%$ under neutral and DTT-free conditions, which increased to more than $38 \%$ upon $10 \mathrm{mM}$ of DTT. When acidic and reduction conditions were simultaneously applied, more than $85 \%$ release of DOX was observed (Ren et al., 2019). For diabetic patients, excessive dosage of insulin would induce severe adverse effects, such as seizures and blindness. Therefore, intelligent carriers with the precise release of insulin according to glucose concentration are imperative. Chen et al. introduced such sense-and-treat carries, with ZIF-8 loaded with GOx and insulin. In the presence of glucose, GOx catalyzed glucose to produce gluconic acid, which triggered the disruption of ZIF-8 and then released insulin for treatment. Meanwhile, encapsulation with GOx and antivascular endothelial growth factor aptamer (VEGF aptamer) simultaneously in this system could be used for macular diseases (Chen et al., 2018c).

\subsection{Other Functional Modification}

Poly(ethylene glycol) (PEG), widely used in most nanomaterials, is applicable to extend drug release, colloidal stability, circulation time, etc. Coating PEG or its composites have been validated for excellent dispersibility, colloidal stability, and long half-lives in Uio-AZB (Roth Stefaniak et al., 2018), Hf-porphyrin MOF (dopamine-derived PEG) (Liu et al., 2018), and Uio-66 (Abanades Lazaro et al., 2017; He et al., 2019b). In addition, in an experiment to deliver drugs by iron-carboxylate MOF (MIL-101-Fe), PEG coating was also able to lengthen the drug release time significantly (Gupta et al., 2019).

Cyclodextrins (CDs) or their derivatives are water-soluble cyclic oligosaccharide with a unique structure of hydrophilic exterior and hydrophobic interior. Anchoring them onto the surface of MOF would endow MOF with the ability to interact with different moieties such as drugs, PEG, and target ligands, thus extending their functions such as controlled drug release and longer half-time. Core-shell NPs, CDs@MIL-100 (Fe), not only retained the virtues of MIL-100 but also extended the applications such as protecting DOX-loaded MIL-100 against aggregation and grafting fluorescent molecules to MIL-100(Fe) (Qiu et al., 2020).

PDA, apart from the application as a photoacoustic (PA) contrast agent, exhibits significant affinity towards interfaces and thus is increasingly considered as a surface functional moiety. PDA-modified MIL-100 was able to improve the dispersibility and stability of NPs except for photothermal conversional efficiency (Zhang et al., 2018e; Feng et al., 2019). Other functional moieties, such as dextran (Lai et al., 2019), lipid (Li et al., 2020b), and glucose (Zhang et al., 2019b), also endowed beneficial effects for MOFs.

\section{THERAPEUTICS}

\subsection{Cancer}

Currently, MOFs are most abundantly used for cancer treatment of all MOF-related nanoformulations. Except for commonly used chemotherapy and radiotherapy, dynamic therapy, immunotherapy, gene therapy, starvation therapy, and combinations are also increasingly being adopted, in which MOFs exert their role as carriers, therapeutic agents, or both (Supplementary Table 1).

\subsubsection{Chemotherapy}

Chemotherapy is still the most routine therapy for cancer, and drugs commonly applied include DOX, 5-fluorouracil (5-FU), cis-platinum, camptothecin (Cam), paclitaxel (PTX), dichloroacetate (DCA), and curcumin (CCM). However, all of these drugs have their limitations, such as poor solubility. MOFs or their composites with various superiorities have been successfully used to deliver these drugs. With high surface area and large porosity, MOFs including ZIF-8 (Tiwari et al., 2017; Dong et al., 2019), MIL-100 (Cabrera-Garcia et al., 2019), and Uio-66 (Gong et al., 2020) have been widely investigated for 
loading of the abovementioned drugs with high loading efficacy. Apart from carriers of bare MOF, various MOF composites were attempted for enhanced chemotherapy. Multifunctional catalytic micromotor ZIF-67/ $\mathrm{Fe}_{3} \mathrm{O}_{4} / \mathrm{DOX}$ constructed by DOX- and $\mathrm{Fe}_{3} \mathrm{O}_{4}$-loaded ZIF-67 displayed up to $58 \%$ drug loading ratio. Furthermore, under hydrogen peroxide $\left(\mathrm{H}_{2} \mathrm{O}_{2}\right)$-based catalytic reaction and external magnetic field, precise delivery and controlled release of DOX could be realized (Wang et al., 2018a). ZIF-8-PAAS nanocomposites by employing PAAS (poly(acrylic acid sodium salt)) as soft templates were successfully used as size-controlled and surface tunable nanodrugs (Yan et al., 2017). Other composites by integrating MOFs with phosphorylcholine-based zwitterionic copolymer for longer circulation time, with carboxymethylcellulose (CMC) biopolymer for oral delivery, were exploited and used for enhanced tumor ablation (Javanbakht et al., 2020; Xie et al., 2020).

To overcome lower anticancer efficacy caused by multiple drug resistance, co-delivery of two or more drugs by MOFs was introduced. Zhang et al. first introduced a multi-drug carrier, PEG-FA/(DOX+VER)@ZIF-8, through encapsulating DOX and verapamil hydrochloride (VER) into ZIF- 8 by one-pot process, followed by modification with PEG-FA. Results showed that drug loading content of DOX and VER was approximately $8.9 \%$ and $32 \%$, and $\mathrm{IC}_{50}$ of PEG-FA/(DOX+VER)@ZIF-8 was much lower than that of free DOX and DOX@ZIF-8 in both MCF7/A and B16F10 cells. Upon modification with PEG-FA, this nanoparticle could be increasingly accumulated in tumors and enhanced the therapeutics (Zhang et al., 2017c). Similarly, ZIF-90 with DOX covalent to surface and 5-FU encapsulation into pores showed that drug loadings were as high as 36.35 and $11-13.5 \mathrm{wt} \%$ for 5FU and DOX, respectively (Zhang et al., 2017a). In another cancer ablation experiment, quercetin (Que) and DOX coloaded to HA/ZIF-8 were proved to synergistically enhance anticancer efficacy through remodeling tumor microenvironment by Que (Li et al., 2019).

\subsubsection{Immunotherapy}

Immunotherapy, based on activating a specific self-immune system, is a burgeoning and promising therapy for cancer. Up to now, as antigen transportation platform for tumor-associated antigens (TAAs) and/or immune adjuvants, MOFs were increasingly used for tumor treatment, where TAAs were delivered to antigen-presenting cells (APCs) to induce $\mathrm{CD} 8^{+}$ cytotoxic $\mathrm{T}$ lymphocyte responses, and adjuvants were used to amplify this response. As carriers, ZIF-8 exhibited high loading of cytosine-phosphate-guanine oligonucleotide (CpG), and the resultant ZIF-8/CpG complexes could induce more cytokine production and thus exerted potent immunostimulatory roles (Zhang et al., 2017b). Amine-functionalized zirconium-based MOF (Uio-AM) was proved to activate the complement systems (Qi et al., 2019), which could become a safe vaccine carrier. Further co-delivery of TAAs and adjuvants CpG ODNs

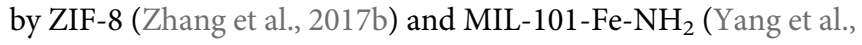
2018b) demonstrated a stronger cellular immune response. Interestingly, another strategy of immunotherapy, by delivery of checkpoint inhibitors nivolumab (NV) by ZIF-8 followed by coating cancer cell membrane, was also demonstrated to be effective for both hematological malignancies and solid tumors (Alsaiari et al., 2021).

\subsubsection{Gene Therapy}

Gene therapy, a personalized therapy based on regulating genes expressed specifically in diseases, has gained more and more attention. Until now, MOFs have been reported successfully for carrying small interfering RNA (siRNA), Cas9/sgRNA compounds, and plasmids. Zhuang et al. have reported a platelet cell membrane-coated ZIF-8 as a siRNA delivery platform, and high silencing efficiency was achieved in vitro. Excitingly, effective tumor targeting and therapeutic efficacy were also observed in in vivo experiments (Zhuang et al., 2020). Similarly, selenium/ruthenium nanoparticles modified MIL$101(\mathrm{Fe})$ loading pooled siRNAs (P-gp siRNA+VEGF siRNA) via surface coordination, which was demonstrated with enhanced cancer ablation (Chen et al., 2017b). Besides, MOF such as ZIF-8 and ZIF-90 have been fascinatingly applied in the delivery of the CRISPR/Cas9 genome editing system, which is a potential direction for disease treatment (Yang et al., 2019; Alyami et al., 2020).

\subsubsection{Dynamic Therapy}

Dynamic therapy, killing cancer cells based on cytotoxic reactive oxygen species (ROS) produced by stimuli such as light, Fenton reaction, and ultrasound (US), includes PDT, chemodynamic therapy (CDT), and sonodynamic therapy (SDT).

\subsubsection{Photodynamic Therapy}

PDT, a noninvasive therapeutic strategy based on light, has been widely used for cancer. After light energy is transferred to PSs, ROS are produced to kill cancer. With higher surface areas, MOFs could deliver large amounts of PSs. In addition, MOFs could be fabricated with PSs as organic linkers. Both strategies could make MOFs conductive in PDT. Until now, various PSs such as porphyrin derivatives (Hynek et al., 2018; Kan et al., 2018), Ce6 (Fu et al., 2020), BODIPY (Wang et al., 2016; Guan et al., 2018), TMPyP4 (Meng et al., 2018), and ZnPc (Xu et al., 2018) have been successfully loaded in different MOFs for PDT. MOFs constructed with porphyrin derivatives as organic linkers were also widely used for PDT (Ma et al., 2017; Zhou et al., 2018b; Gao et al., 2019). For example, the Ce6 delivery system based on ZIF-8 with modification with HA exhibited ultrahigh Ce6 encapsulation capability (76.8\%). And this nanoparticle killed about $88.4 \%$ of cancer cells upon irradiation (Fu et al., 2020). Furthermore, without any drug loading, $\mathrm{Sm}-\mathrm{H}_{2} \mathrm{TCPP}$ themselves synthesized by $\mathrm{Sm}^{3+}$ as metal nodes and PSs tetrakis (4-carboxyphenyl)porphyrin $\left(\mathrm{H}_{2} \mathrm{TCPP}\right)$, as organic linkers were demonstrated with prominent ROS generation capacity (Gao et al., 2019).

Considering that the hypoxic microenvironment contributes to the unsatisfactory effect of PDT, various strategies, including producing extra oxygen $\left(\mathrm{O}_{2}\right)$, reducing the $\mathrm{O}_{2}$ consumption, and decreasing the GSH levels, were conducted to improve PDT efficiency. Core-shell nanosystem RC@TFC, with RAP-Ce6 composites as core and CAT-loaded Fe-MOF as shell, could improve hypoxia environment through catalyzing abundant 
$\mathrm{H}_{2} \mathrm{O}_{2}$ to $\mathrm{O}_{2}$ by CAT and inhibiting expression of hypoxiainducible factor-1a by rapamycin simultaneously, thus potentiating the PDT (Liu et al., 2019b). Other $\mathrm{O}_{2}$-evolving approaches such as MOF-nanozyme composites, including Au@ZIF-8 (Ma et al., 2019) and PCN-224-Pt (Zhang et al., 2018d) nanostructures, were also able to overcome the hypoxic conditions and improve the PDT efficiency. In contrast, to improve the $\mathrm{O}_{2}$ supply, Chen et al. introduced another ZIF/PC complex, in which mitochondrial complex I inhibitor papaverine (PPV) was encapsulated and delivered to the tumor to reduce the intratumor oxygen consumption (Chen et al., 2021). As highlevel GSH would also reduce the PDT effect, MOF-2 was designed with $\mathrm{Cu}(\mathrm{II})$ as an active center to absorb and lower GSH and then enhanced PDT (Zhang et al., 2018c).

Apart from the hypoxic environment, the penetration depth is another obstacle that limits the application of PDT. Shi et al. developed a nanosystem by decorating titanium dioxide nanoparticles on upconversion nanoparticle-MOF composites. Upon irradiation by $980-\mathrm{nm}$ NIR light, upconversion nanoparticles could generate ultraviolet and visible lights and induce titanium dioxide and porphyrin to produce ROS through Type I and II PDT. More importantly, this nanosystem could realize deep tissue penetration (Shi et al., 2020).

\subsubsection{Chemodynamic Therapy}

CDT, an emerging therapeutic approach based on tumor environment, is a kind of treatment based on in situ production of hydroxyl radical $(\cdot \mathrm{OH})$ via Fenton reaction and Fenton-like reaction. In CDT, transition metal ions such as $\mathrm{Fe}^{2+}$, $\mathrm{Cu}^{2+}$, and $\mathrm{Mn}^{2+}$ catalyze $\mathrm{H}_{2} \mathrm{O}_{2}$ to $\cdot \mathrm{OH}$ and kill tumors. Therefore, MOFs constructed by these metal ions could be used for CDT. Moreover, increased $\mathrm{H}_{2} \mathrm{O}_{2}$ levels and decreased GSH levels are also beneficial for CDT. Wu et al. first introduced an autocatalytic nanoreactor through coating CuMOF on GOx-loaded dendritic mesoporous organosilica nanoparticles (DMONs). Once entering cells, CuMOF was disrupted in acidic endo/lysosomes. On the one hand, GOx catalyzed glucose to generate $\mathrm{H}_{2} \mathrm{O}_{2}$; on the other hand, $\mathrm{Cu}^{2+}$ induced $\mathrm{GSH}$ depletion and reduced $\mathrm{Cu}^{2+}$ to $\mathrm{Cu}^{+}$, both of which were important for self-boosting CDT (Wu et al., 2020). Other approaches without transition metal were also acceptable for CDT. By embedding horseradish peroxidase (HRP) and GOx into ZIF-8, a cascade catalytic bioreactor was reported against the solid tumor. After degradation of ZIF-8 in acidic conditions of cancer, HRP and GOx were released. Released GOx catalyzed glucose to produce $\mathrm{H}_{2} \mathrm{O}_{2}$, which was further catalyzed by HRP to $\mathrm{OH}$ (Bai et al., 2019). NanozymeMOF complex, for example, PEG-modified Cu-Pd@MIL101(CPMP) with high POD-like and SOD-like activities, as well as the ability of depletion of GSH could produce a high level of hydroxyl radicals and thus be effective for tumor treatment (Yang et al., 2021b).

\subsubsection{Sonodynamic Therapy}

SDT is a burgeoning and promising noninvasive therapy due to its superiority of deeper penetration. Under low-intensity US and sonosensitizers, ROS are produced to kill cancer. As in PDT, by loading sonosensitizers such as Ce6 in MOF, SDT for cancer ablation could be achieved under US (An et al,, 2020a). In addition, MOF based on active components harboring the capacity of sonosensitizers including porphyrin derivatives (Xu et al., 2021) and Ti (Liang et al., 2021) could also be used for SDT.

\subsubsection{Others}

Photothermal therapy (PTT), another noninvasive phototherapy, exerts its function through converting light energy to thermal energy, in which photothermal agents (PTAs) play an essential role during the progress (Hussein et al., 2018). The role of MOFs in PTT is mainly as carriers of PTA or as PTA.

As a widely used carrier, ZIF-8 has been also reported for successful loading autophagy inhibitor for tumor treatment through the autophagy pathway (Chen et al., 2018e). Both in vitro and in vivo experiments showed that 3-methyladenine (3-MA)-loaded MOFs could effectively inhibit the tumor as compared with free 3-MA.

Ferroptotic therapy, a novel mechanism of cell death through regulating different pathways such as lipid peroxide, has also attracted increasing attention in antitumor. As this therapeutic modality is dependent on iron ions, MOFs based on $\mathrm{Fe}$ or Feincluding could enhance the sensitivity of ferroptosis. By combining Fe-MOF with ferroptosis inducer, the efficacy of the antitumor would be enhanced (Liu et al., 2021b; Xin et al., 2021).

\subsubsection{Combination Therapy}

Compared with monotherapy, which often fails to achieve a desirable therapeutic effect, combination therapies may be more effective. Currently, various combination therapies such as chemotherapy/PDT (Liu et al., 2018; Ren et al., 2020), PDT/ PTT (Gao et al., 2018a; Wang et al., 2019c), PDT/CDT (Wu et al., 2021a), and PDT/immunotherapy (Lan et al., 2018; Cai et al., 2020; Ni et al., 2020) have been increasingly applied. In this way, MOFs deliver different functional agents to kill tumors in different ways. pH-responsive nanoprobes, AuNCs@MOFDOX, were constructed by loading PS gold nanoclusters (AuNCs) and DOX into ZIF-8 for simultaneous chemotherapy/PDT. In the tumor acidic conditions, DOX and AuNCs were released. Under irradiation, a large amount of singlet oxygen was produced and further enhanced the chemotherapy. Excitingly, compared with a single therapy, this combination completely eliminated the tumor in an in vivo experiment (Zhang et al., 2020b). In another multifunctional core-shell nano-agent (ZDZP@PP), PS protoporphyrin IX (PpIX)-loaded ZIF-8 was grown on the ZIF-67/DOX followed by PDA-PEG coating. Once internalized by cancer cells, PDA and ZIF-8 were disrupted with PpIX and ZIF-67 released. ZIF-67 as an $\mathrm{H}_{2} \mathrm{O}_{2}$ catalyst catalyzed $\mathrm{H}_{2} \mathrm{O}_{2}$ to oxygen and improved the PDT. Meanwhile, DOX from ZIF-67 killed cancer cells and thus realized oxygen self-sufficient chemotherapy/PDT combination (Ren et al., 2020) (Figure 5). Compared with MOFs only used as carriers, active moiety-containing MOFs such as PSs-MOF could potentiate the effect of treatment. For instance, hypoxia-activated prodrug (tirapazamine (TPZ))-loaded Hf-porphyrin MOFs have high porphyrin loading and produced higher ROS for PDT, 


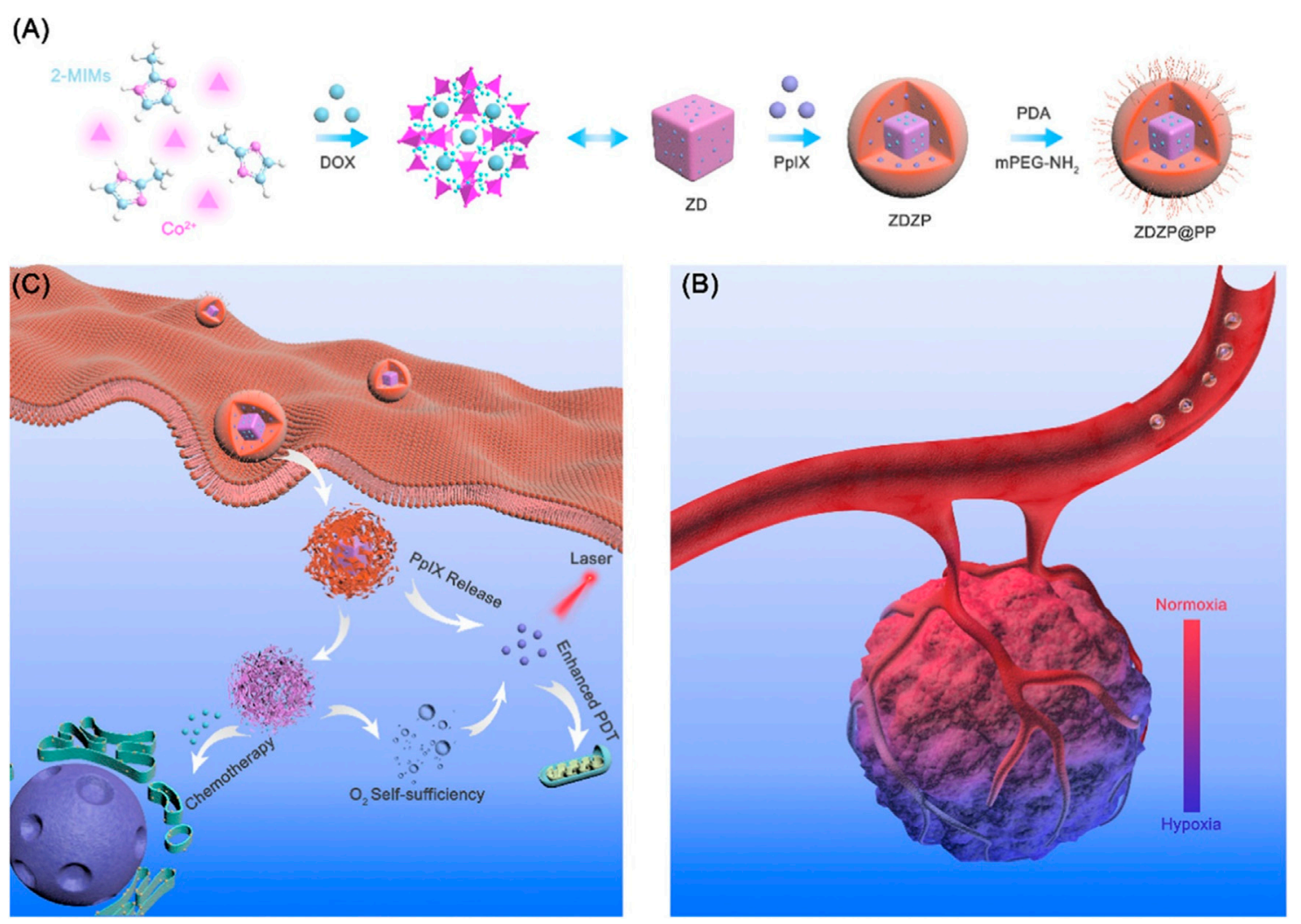

FIGURE 5| Schematic of ZDZP@PP NPs in photodynamic therapy (PDT)/chemotherapy combination therapy for tumor. (A) Synthesis of ZDZP@PP Nanoparticles. (B,C) Schematic illustration of ZDZP@PP in combination therapy. Reproduced with permission. Copyright 2020, ACS Applied Materials \& Interfaces.

which depleted oxygen and induced activation of TPZ for enhanced PDT/chemotherapy (Liu et al., 2018).

In addition, PDT/PTT is also the most common therapy. By gas absorption and modifiable properties, a biomimetic $\mathrm{O}_{2-}$ evolving nanoplatform was fabricated. In this system, $\mathrm{O}_{2^{-}}$ loaded Uio-66 was conjugated with ICG as both PSs and PTAs, followed by RBC membrane coating. Upon irradiation, the photothermal property of ICG facilitated $\mathrm{O}_{2}$ release and then enhanced the PDT/PTT (Gao et al., 2018a). In another exciting experiment for tumor ablation, nano-agents (Zn-TCPP) constructed by $\mathrm{Zn}^{2+}$ as metal nodes and TCPP as organic linkers were used as PTAs more often than PSs, which benefited from large $\pi$ electron conjugated in aromatic macrocycle (Wang et al., 2019c).

\subsection{Inflammatory Diseases}

In most recent years, researchers have seen the germination and development of MOFs in inflammation-related diseases. In summary, the main applications of MOFs in inflammation or inflammatory conditions are as follows: 1) the inherent therapeutics of MOFs, 2) MOFs as delivery systems or active carriers of drugs and antioxidant enzymes, and 3) nanozymes based on MOFs (Supplementary Table S2).

MOFs exert their role in inflammatory disease mainly as drug carriers. Supramolecular $\gamma$-CD-MOFs as carriers of sodium diclofenac (DFNa) showed approximately 50\% entrapment efficiency and more prolonged controlled release of drugs. In carrageenan-induced mouse paw edema, the inhibition rate was 88.7\% after treatment with $\gamma$-FeCD-MOF (Abucafy et al., 2018). As for microenvironment and pathogenesis, Xiong et al. engineered an intelligent $\mathrm{pH}$-responsive drug delivery system modified by hydrophilic agent HA, which could improve the hydrophilicity of MOFs and inflammation of OA simultaneously. In the weak acidic environment of osteoarthritis (OA), MIL$100(\mathrm{Fe})$ was disrupted, and protocatechuic acid (PCA) was released for alleviating inflammation by reducing the inflammatory mediators (Xiong et al., 2020). Similarly, as mentioned above, an oxidation-responsive MOF, Ce-MOF@ PSS, was designed based on the microenvironment of inflammatory bowel disease (IBD) and antioxidant ability of $\mathrm{Ce}^{3+} / \mathrm{Ce}^{4+}$. By utilizing the negatively charged properties of poly(sodium-4-styrenesulfonate) (PSS), the obtained CeMOF@PSS adhered to the inflammatory intestine. In inflammatory sites, the reaction between $\mathrm{ROS}$ and $\mathrm{Ce}^{4+}$ eliminated ROS and induced the pore size transformation of Ce-MOF, thus releasing drugs. Apart from the excellent performance of inflammation-targeting ability in different colitis models, significant alleviation of inflammation was observed in spontaneous colitis (Yin et al., 2021).

Antioxidant enzymes, with the capacity of catalyzing cytotoxic free radicals to nontoxic ingredients, are also effective in inflammatory diseases. Using natural enzymes including 


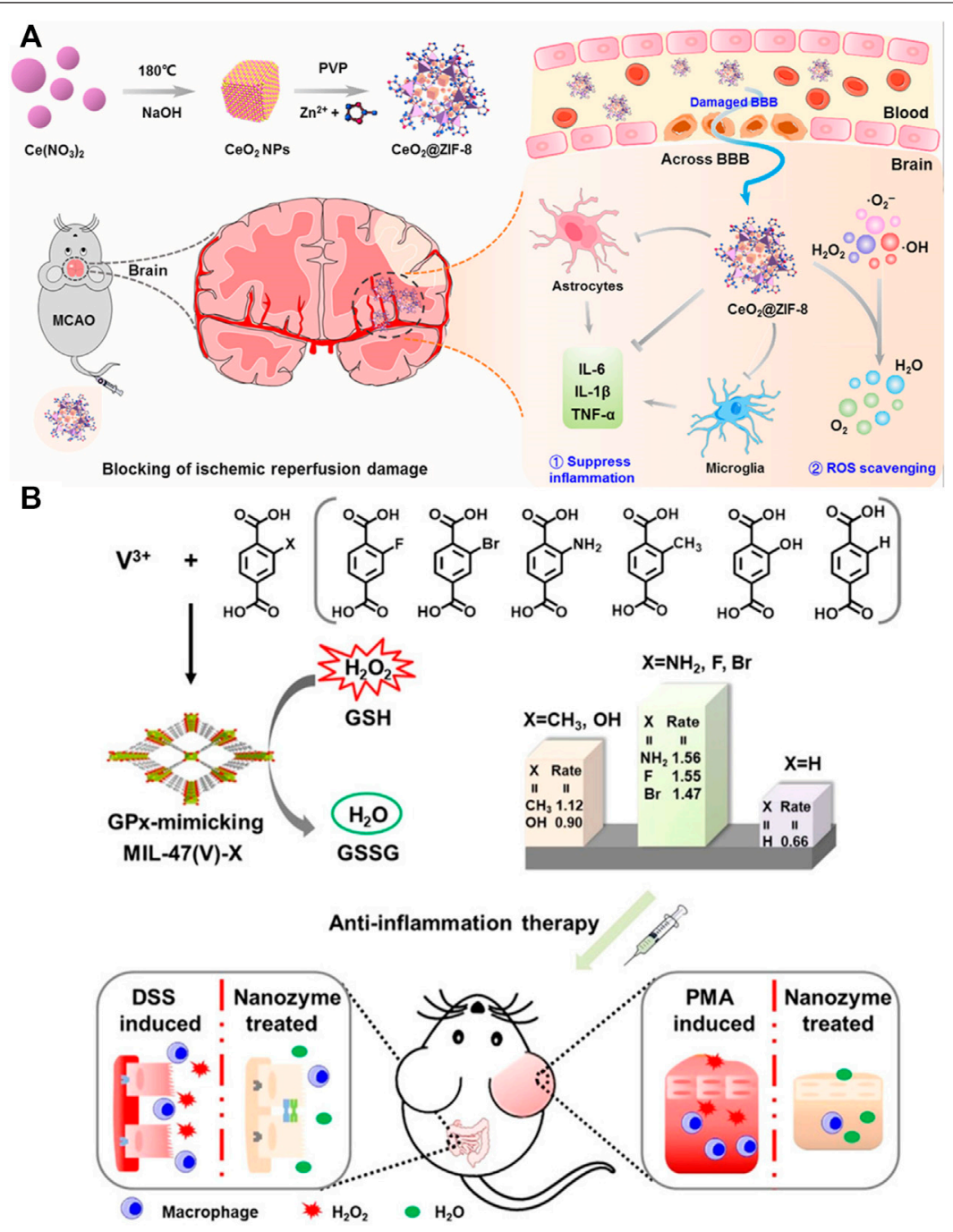

FIGURE 6 | Schematic of nanozyme based on metal-organic frameworks (MOFs) for inflammatory diseases treatment. (A) Schematic of CeO $@$ @ZIF-8 synthesis and application mechanism against ischemic stroke. Reproduced with permission. Copyright 2020, Science Advances. (B) Schematic of designing principle and application in the anti-inflammatory treatment of GPx-mimicking MIL-47(V)-X-MOF nanozymes. Reproduced with permission. Copyright 2021, Angewandte Chemie International Edition.

superoxide dismutase (SOD) and CAT into PCN-333(Al) was proved to protect cells from toxic ROS for up to a week as compared with free enzymes with only a short duration, which have the potential for treating inflammation (Lian et al., 2017). Similarly, He et al. constructed bioactive ZIF-8-capped ceria NPs ( $\mathrm{CeO}_{2} @ Z I F-8$ NPs) for reperfusion-induced injury in ischemic stroke. As expected, these NPs effectively prevented and improved ischemic stroke by inhibiting lipid peroxidation and reducing oxidative damage and neuron apoptosis (He et al., 2020) (Figure 6A). Wu et al. further introduced a glutathione peroxidase (GPx) mimicking MIL-47 (V)- $\mathrm{NH}_{2}$ nanozyme based on MIL-47 (V) through ligand engineering strategy. Both in vitro and in vivo experiments demonstrated excellent antioxidant capacity, which provided a new direction for inflammation treatment (Wu et al., 2021b) (Figure 6B). More interestingly, $\mathrm{Mg} / \mathrm{COOH}-\mathrm{MOF}$ without carrying any other functional agents showed excellent anti-inflammatory activity and is beneficial for OA treatment (Li et al., 2020c).

In a recent study for managing severe sepsis, Liu et al. designed a cationic MOF by grafting cationic polyethylenimine (PEI) to ZIF-8. As a "nanotrap," this MOF could scavenge circulation cell-free DNA (cfDNA) produced by damaged cells and inhibit cfDNA-induced TLR activation and other signaling pathways, thus alleviating the inflammation in sepsis, which provided a novel strategy for inflammation treatment (Liu et al., 2021a).

\subsection{Antibacteria}

Bacterial infection has become a public burden, which causes increased mortality and morbidity worldwide, with inadequate treatment induced by antibiotics abuse. Hence, it is crucial to seek newer and more effective ways for bacterial eradication. 


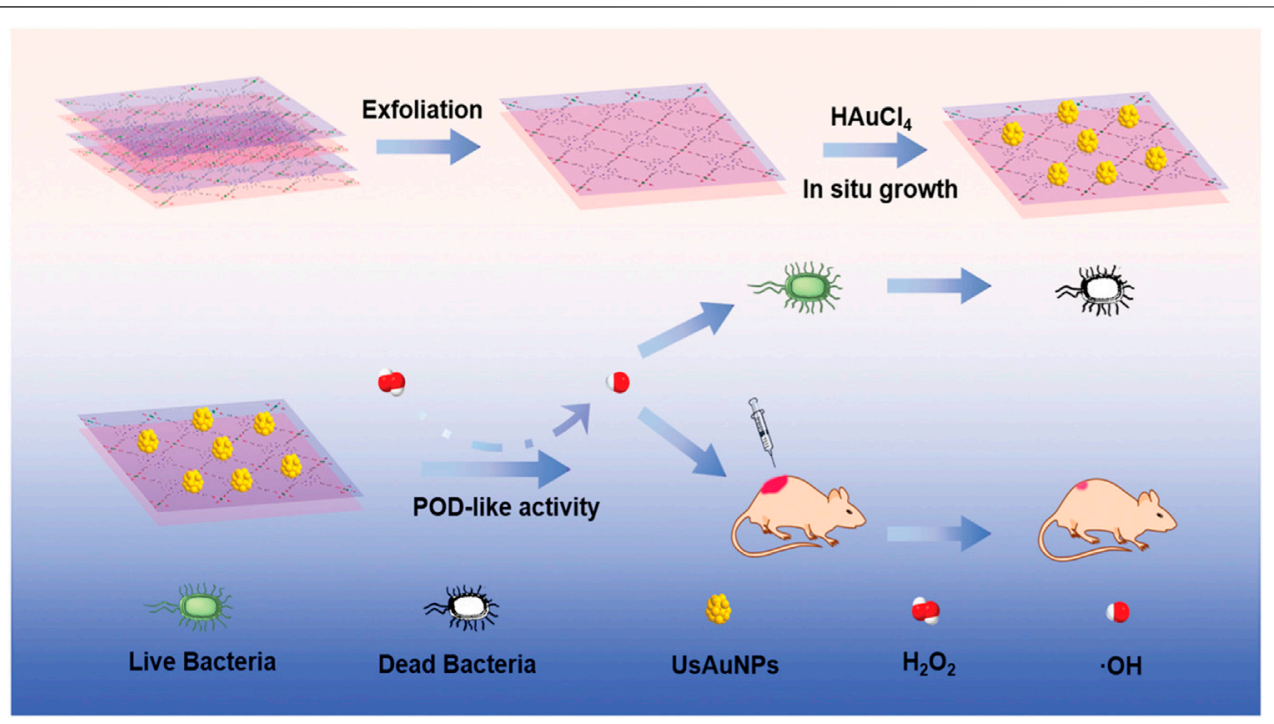

FIGURE 7 | Schematic of UsAuNP/metal-organic framework (MOF) hybrid with POD-like activity for antibacterial therapy. Reproduced with permission. Copyright 2020, Small.

Drugs, especially antibiotics, are still the main therapeutics. However, the hydrophobic nature, frequent demonstration, and the resultant drug resistance reduce the effect of drugs. MOFs as carriers of antibiotics have solved these problems. Until now, vancomycin (Van) (Chowdhuri et al., 2017), ceftazidime (Sava Gallis et al., 2019), ciprofloxacin (CIP) (Esfahanian et al., 2019), tetracycline (Tet) (Zhang et al., 2019d), and rifampicin (Ahmed et al., 2020) have been successfully encapsulated into ZIF-8 and its composites for antibacterials with intelligent drug release. For example, ceftazidime@ZIF-8 particles by encapsulating antibiotics into ZIF-8 showed sustained release of ceftazidime for up to a week, and $50 \mu \mathrm{g} / \mathrm{ml}$ of nanoparticles inhibited Gram-negative Escherichia coli completely (Sava Gallis et al., 2019). Interestingly, in another delivery system, zinc ions released from ZIF-8 under an acidic environment exhibited a synergistic antibacterial effect (Zhang et al., 2019d). Compared with loading antibiotics, Mao et al. designed a precise antibacterial system by perfusing metabolic labeling molecule 3-azido- $d$-alanine (d-AzAla) into MIL$100(\mathrm{Fe})$. Under $\mathrm{H}_{2} \mathrm{O}_{2}$ condition, $d$-AzAla was released and selectively adhered to the cell walls of bacteria, which recognized and reacted with additional dibenzocyclooctyne (DBCO)-modified PS NPs, thus achieving precise bacteria killing by selective FL labeling (Mao et al., 2018a).

The application of silver nanoparticles (AgNPs), another promising antibacterial approach, is hampered by their aggregation. Immobilization of AgNPs into $\gamma$-cyclodextrin MOFs (CD-MOFs) (Shakya et al., 2019; Luo et al., 2020) and Ag-doped magnetic MOF ( $\gamma$ - $\mathrm{Fe}_{2} \mathrm{O}_{3} @ \mathrm{SiO}_{2} @ \mathrm{ZIF}-8$-Ag) (Rahmati et al., 2020) showed superior performance in bacterial inhibition. In addition, nanozyme-based AuNP/MOF hybrid with excellent POD-like activity exhibited significant antibacterial effects ( $\mathrm{Hu}$ et al., 2020) (Figure 7). Li et al. constructed self-activated cascade MOF/enzyme composites with peroxidase-like activity based on GOx-loaded MIL-MOF, where GOx could reduce the $\mathrm{pH}$ value for optimum activity of MIL to inhibit the methicillin-resistant Staphylococcus aureus (MRSA) (Li et al., 2020a). In combination therapy, for example, ZIF-8@PDA composites as carriers of Van have been demonstrated to have effective bacterial eradication through NIR/pH-responsive synergistic PTT/chemotherapy (Xiao et al., 2021). Another composite nanomaterial, ZIF-8PAA-MB@AgNPs@Van-PEG, exhibited an ability against three kinds of bacteria based on $\mathrm{PDT} / \mathrm{AgNP} /$ chemotherapy (Chen et al., 2019a) (Figure 8).

In addition, as surface modification agents, MOFs incorporated into matrix materials such as CS are used to enhance the antibacterial properties. For example, Uio-66- $\mathrm{NH}_{2} / \mathrm{CS}$ composite by fusing $\mathrm{UiO}-66-\mathrm{NH}_{2}$ with $\mathrm{CS}$ has peroxidase and oxidase mimicking activity and exhibited good antibacterial properties in both $\mathrm{H}_{2} \mathrm{O}_{2}$-present and $\mathrm{H}_{2} \mathrm{O}_{2}$-absent conditions. In the presence of $\mathrm{H}_{2} \mathrm{O}_{2}$, this composite killed bacteria through peroxidase-mimicking activity; however, without $\mathrm{H}_{2} \mathrm{O}_{2}$, it could also degerm under $30 \mathrm{~min}$ of UV pre-irradiation (Wang et al., 2021).

\section{IMAGING}

Early diagnosis and monitoring of diseases by imaging technology are indispensable for timely precaution and treatment. Current imaging technologies include MRI, CT, optical imaging, PA imaging (PAI), and positron emission tomography (PET). MOFs as contrast agents or imaging agent carriers have been widely applied in imaging for tracing diagnosis or therapeutics (Supplementary Table S3).

\subsection{Magnetic Resonance Imaging}

MRI is a noninvasive image modality, which is based on the interaction between water proton nuclei of samples and external magnetic field (Foltz and Jaffray, 2012), in which contrast agents 


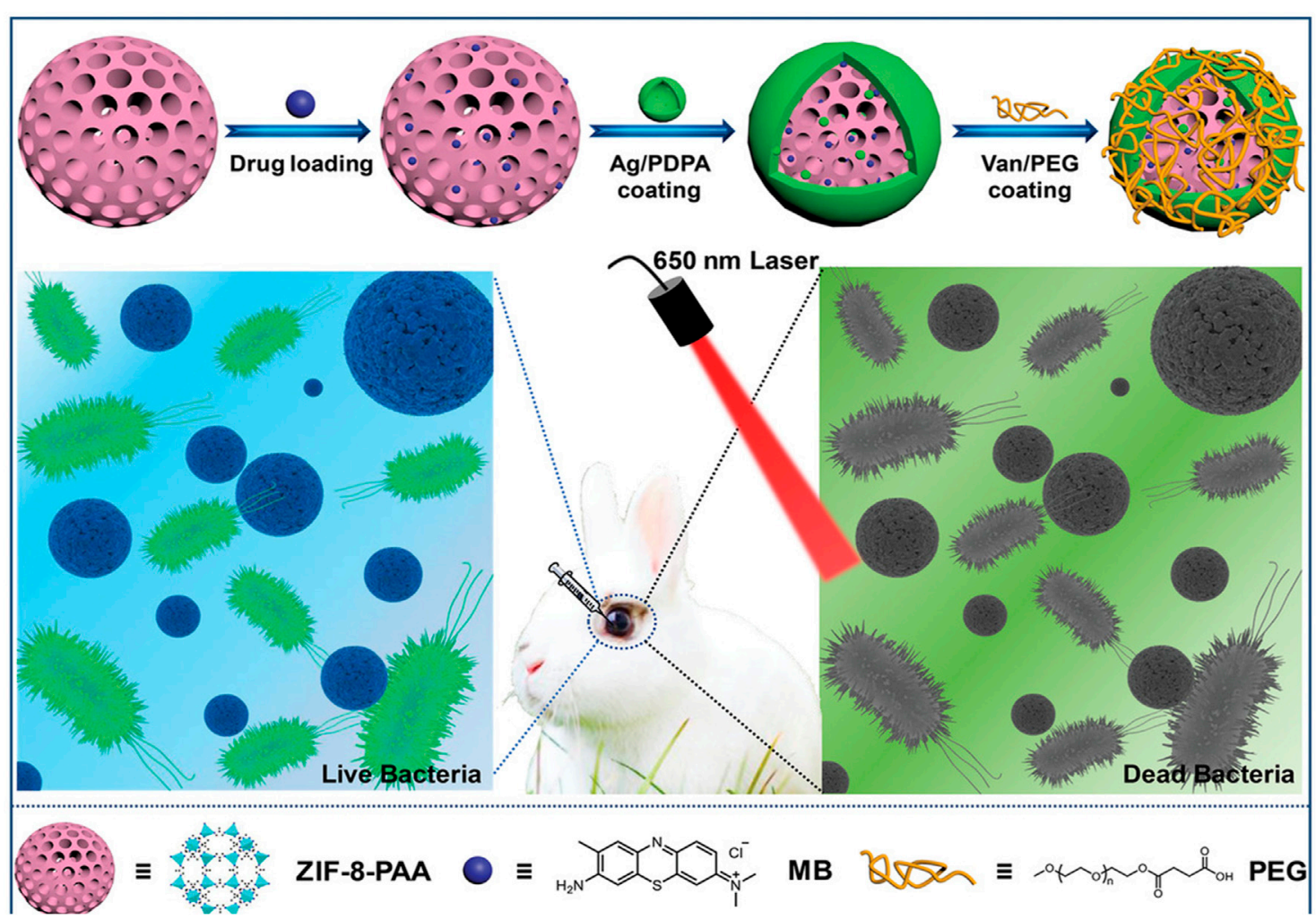

FIGURE 8 | Schematic of ZPMAVP system for application in synergistic chemotherapy and photodynamic therapy for endophthalmitis. Reproduced with permission. Copyright 2019, Small.

amplify the signal by enhancing the relaxivities of water protons. Magnetic metal ions such as $\mathrm{Fe}, \mathrm{Mn}$, and Gd are widely used as contrast agents. Among them, $\mathrm{Gd}$ and $\mathrm{Mn}$ are used as $\mathrm{T}_{1^{-}}$ weighted-enhanced agents, while $\mathrm{Fe}$ was used as $\mathrm{T}_{2}$-weighted MRI agents (Xiao et al., 2016). However, disadvantages such as low retention time and toxicity induced by frequent dosing of single metal significantly impede their application. With ideal size, high loading efficiency, and ease of modification, MOFs constructed with the above metal as nodes or with the above metal oxides loaded or as coating could be used for increasing retention time and relaxivities. For example, $\mathrm{Fe}_{3} \mathrm{O}_{4} @ \mathrm{UiO}-66$ composites, fabricated by growing $\mathrm{UiO}-66$ on $\mathrm{Fe}_{3} \mathrm{O}_{4}$, exhibited good capability as contrast agents with transverse relaxivity ( $\mathrm{r} 2)$ of $1,396 \mathrm{mg} \mathrm{mL}^{-1} \mathrm{~s}^{-1}$, which was much higher than that of commercial Fe-based agents such as Resovist $\left(150 \mathrm{nM}^{-1} \mathrm{~s}^{-1}\right)$ (Zhao et al., 2016). Other Fe-MOFs such as MIL family (MIL53, MIL-100, etc.), Mn-MOF such as Mn-porphyrin MOF (PCN222) (Zhang et al., 2018a; He et al., 2019a), $\mathrm{MnO}_{2}$-coated porphyrin-MOF (PCN224) (Zhang et al., 2019a; Min et al., 2019; Tian et al., 2019), and MOFs containing $\mathrm{Mn}^{2+}$ (Meng et al., 2021) also showed beneficial performance for MRI.

\subsection{Computed Tomography}

CT image is a common technology of diagnosis and treatment by visualizing the internal structures of the body, which is based on the X-ray attenuation after tissue absorption. The addition of contrast agents would enhance the contrast between lesion tissue and normal tissues. Apart from barium sulfate suspensions only applied in CT for the gastrointestinal (GI) tract, iodinated aromatic molecules are routine contrast agents for CT images. Still, they also cause short blood circulation and poor sensitivity (Lee et al., 2013). CT contrast agent Uio-PDT, formed by diiodosubstituted monocarboxyl-functionalized BODIPY ( $\left.\mathrm{I}_{2}-\mathrm{BDP}\right)$ with metal, not only overcame the limitation of iodinated molecules but also exhibited the best imaging performance after $24 \mathrm{~h}$ (Zhang et al., 2017d). Other high-Z elements, including $\mathrm{Zr}$ - and Hf-based MOFs, have also been evaluated as contrast agents for CT (Dekrafft et al., 2012).

\subsection{Positron Emission Tomography}

$\mathrm{PET}$ is an imaging technology for monitoring metabolism processes by tracing signals from radioisotopes $\left({ }^{89} \mathrm{Zr},{ }^{64} \mathrm{Cu}\right.$, ${ }^{68} \mathrm{Ga}$, etc.) doped into contrast agents (Derlin et al., 2018). Therefore, MOFs based on these metals with targeting modification would be beneficial for PET imaging. By incorporating positron-emitting isotope ${ }^{89} \mathrm{Zr}$ into Uio-66 MOF, combining with PEG modification, and targeting peptide ligand (F3), Chen et al. examined the distribution and clearance profile of MOF in vivo. Both in vivo and ex vivo PET imaging results showed that tumor uptake of ${ }^{89} \mathrm{Zr}$-UiO-66 was faster and higher than in all other major organs, which exhibited potential tumor contrast (Chen et al., 2017a). 


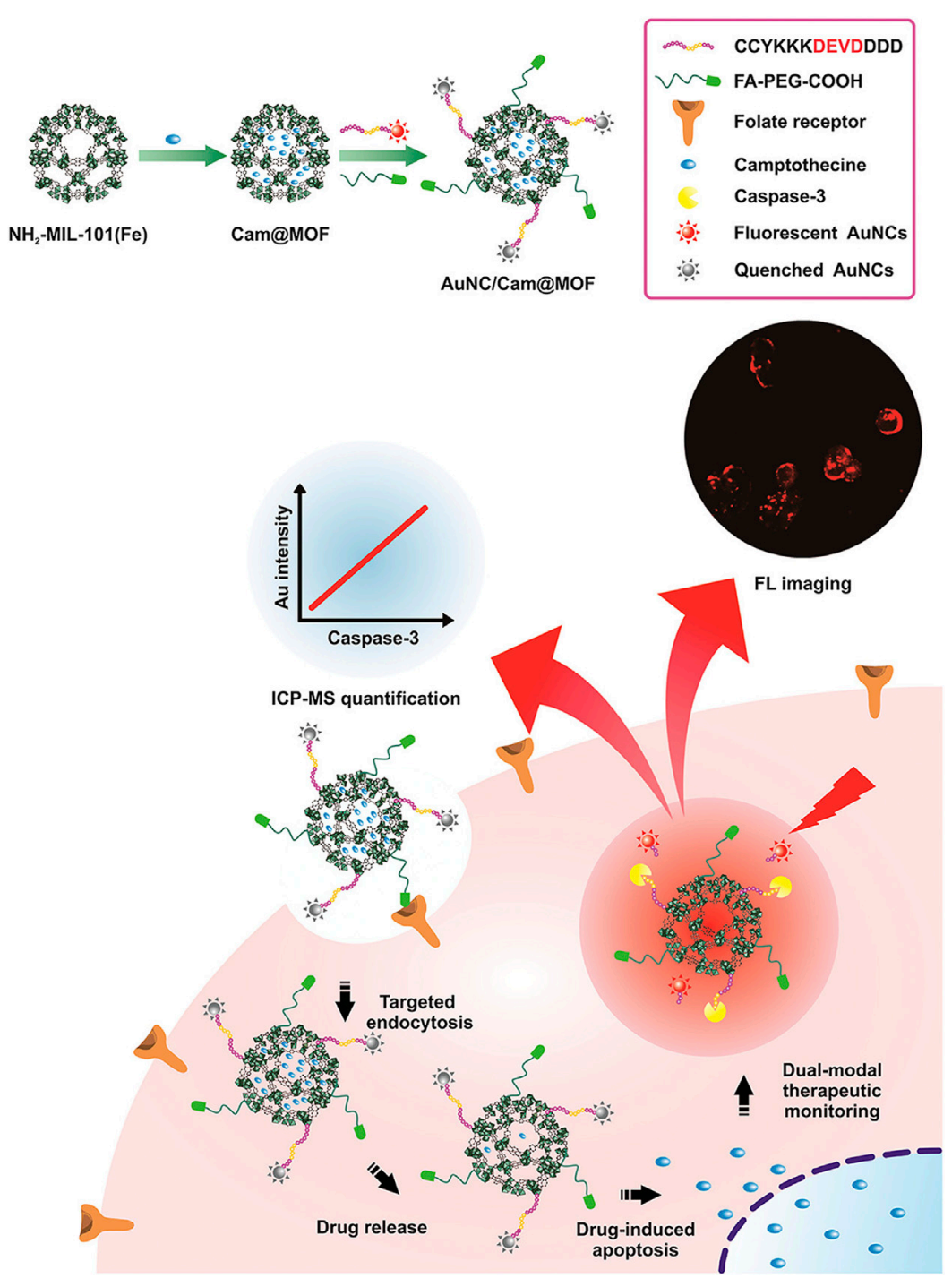

FIGURE 9 | Diagram of construction of multifunctional AuNC/Cam@MOF nanoprobe and its applications including fluorescence (FL) imaging. Reproduced with permission. Copyright 2019, Analytical Chemistry.

\subsection{Optical Imaging}

Optical imaging provides opportunities for real-time monitoring of tissues at the microscopic level without tissue damage, including FL, phosphorescence, and chemiluminescence (CL) imaging (Pirovano et al., 2020). In optical imaging, FL is the most widely used. MOFs constructed with luminophore as metal nodes or organic linkers, loaded with FL dyes, and combined with other functional moieties including quantum dots (Qin et al., 2020), AuNPs (Yin et al., 2019), ruthenium(II)tris(bipyridyl) cationic complex (Ru (bpy) ${ }_{3}{ }^{2+}$ ) (Chen et al., 2017c) have been successfully applied. For example, Cy@ZIF-8, by incorporating NIR dye cyanine (Cy) to ZIF-8, not only improved the water solubility of Cy but also exhibited strong FL both in vitro and in vivo (Li et al., 2018). Yin et al. constructed a nanoplatform for real-time monitoring and quantifying the therapeutic drug response. In this system, fluorescent AuNCs were decorated onto Cam@MIL-101 (Fe) through a peptide, which could be cleaved by intermediate products of camptothecin. Once internalized by HepG2 cells, Cam induced the production of caspase-3, thus releasing AuNCs and restoring their FL. Meanwhile, released Au could be used to quantify the amount of caspase-3 (Yin et al., 2019) (Figure 9).

\subsection{Photoacoustic Imaging}

PAI has the superiorities of superb contrast, super spatial resolution, and deeper penetration based on the optoacoustic effect and contrast agent (Fu et al., 2019). Gold nanoparticles, dyes with NIR absorption including indocyanine green (ICG), and porphyrin derivatives were widely used for PA. The combination of these dyes with MOFs may enhance their performance. By integrating $\mathrm{Au}$ nanostar with ZIF-8, Deng et al. successfully fabricated a novel light-responsive yolk-shell 

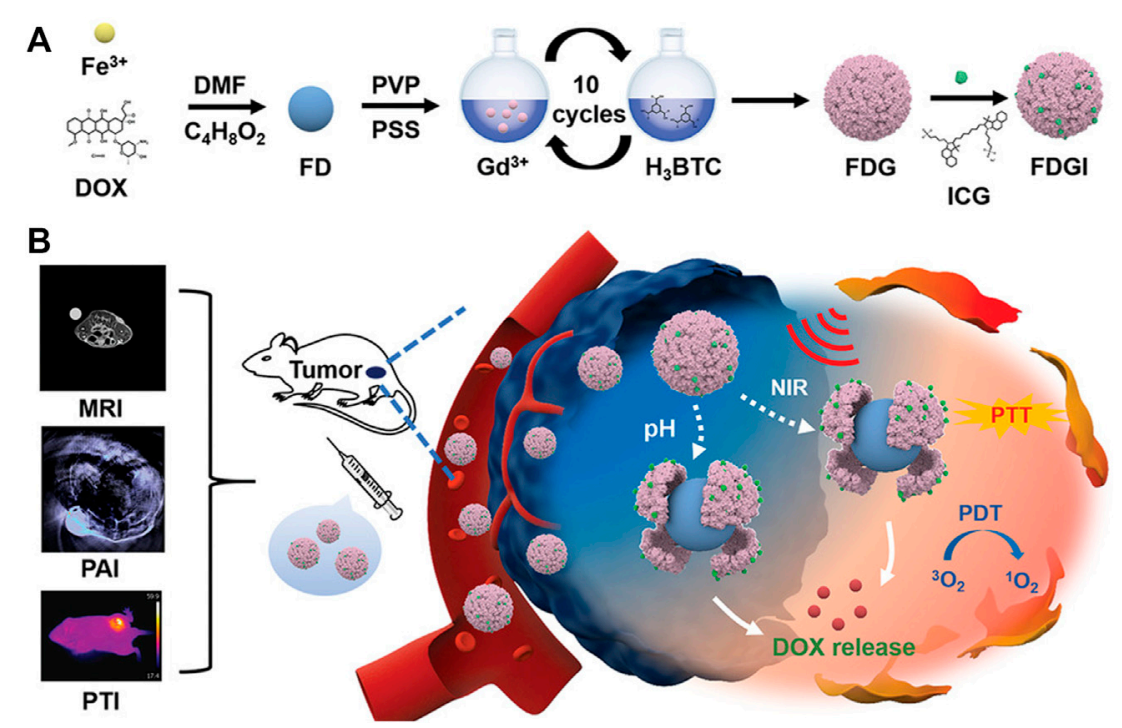

FIGURE 10 | The synthesis and application of Fe-DOX@Gd-MOF-ICG nanoplatform (FDGI NPs) in MR/photoacoustic/photothermal (MR/PA/PT) multimodal imaging-guided therapeutics. (A) The synthesis scheme of FDGI NPs. (B) The application of FDGI NPs in MR/PA/PT imaging-guided therapeutics. Reproduced with permission. Copyright 2020, Advanced Healthcare Materials.

nanosystem. Upon 1,064 nm (NIR-II bio-window) of laser irradiation, this nanosystem showed excellent photothermal conversion capability for PTT. In addition, with intense NIR absorbance, the potential of PAI was performed in vivo. Compared with weak signals from control, tumor sites of mice administrated with Au@ZIF-8 exhibited strong PAI signals at $12 \mathrm{~h}$ (Deng et al., 2019). In another experiment, ICG-engineering MIL$100(\mathrm{Fe})$ by integrating MIL-100 with ICG showed strong NIR absorbance. In addition, the solubility and targeting ability of ICG were improved. As expected, both in vitro and in vivo experiments demonstrated satisfactory results in PAI (Cai et al., 2017).

\subsection{Multi-Modal Imaging}

As none of these image modalities are fully qualified for disease monitoring due to the limitation of each modality, combining them with two or more technologies would be a better choice. For instance, in bioactive Fe-DOX@Gd-MOF-ICG nanoplatform, the Gd-MOF shell was used for MRI apart from the protective shell for Fe-DOX cores. Besides, both PAI and photothermal imaging (PTI) were successfully introduced by loading ICG, thus realizing the multimodal imagingguided therapeutics (Zhu et al., 2020) (Figure 10). Other MOFs without loading dyes and modification could also achieve multimodal imaging. For example, MOFs synthesized by integrating $\mathrm{Ga}^{3+}$ with fluorescent linker pDBI (pDBI $=1,4$-bis(5-carboxy$1 H$-benzimidazole-2yl)benzene) without any other modification could be used for FLI and MRI simultaneously (Kundu et al., 2016).

\section{THERANOSTICS}

Disease theranostics, a combination of therapeutics and diagnostics, has attracted increasing attention in personalized and accurate treatment for diseases, which simultaneously require the diagnosis and monitoring for the treatment processes.

Functional MOFs constructed by metals such as $\mathrm{Hf}, \mathrm{Fe}, \mathrm{Cu}$, and Gd or/and organic linkers such as porphyrins could deliver therapeutic agents selectively to lesion sites with surface modification and thus quickly realize imaging-guided treatment. For example, the Hf-porphyrin platform with hypoxia-activated prodrug (TPZ) trapped and modified with dopamine-derived polymer (DOPA-PIMA-mPEG) was successfully used in CT imaging-assisted PDT/chemotherapy. In this system, by incorporating porphyrin into a platform, the efficiency of PDT was improved. Then, TPZ was activated under hypoxic conditions induced by PDT and further enhanced the treatment. Furthermore, after being intratumorally injected, an unmistakable enhanced CT signal was observed at tumor sites. Another "all-in-one" nano-agent based on Hf as metal cluster and Mn (III)-porphyrin as linkers with FA coating exhibited excellent performance both in treatment and in imaging. On the one hand, FA guided nano-agent to tumor sites, and then the CAT-like activity of $\mathrm{Mn}$-porphyrin improved the hypoxic condition by catalyzing $\mathrm{H}_{2} \mathrm{O}_{2}$ to $\mathrm{O}_{2}$, which boosted the PTT/RT combination therapy. On the other hand, this system has high performance for MRI/CT/PAI enhancements. Signals from CT and MR were increased by 1.7 -fold and 3 - to 5 -fold, respectively. Thus, this triple-modality imaging-assisted PTT/RT system showed high potential in tumor theranostics (Bao et al., 2020).

Similarly, MOFs as carriers could also realize multifunctional theranostics. Guo et al. engineered a $\mathrm{pH} / \mathrm{NIR}$ dual-responsive nanocarrier based on ZIF-8 for MRI/PAI-aided chemophototherapy. In this system, DOX was embedded into ZIF-8 through a one-pot way, followed by modification with PDA, Mn ions, and PEG. Under NIR laser irradiation, the resultant ZIF-8/ DMPP showed a satisfactory thermal effect, which accelerated the 


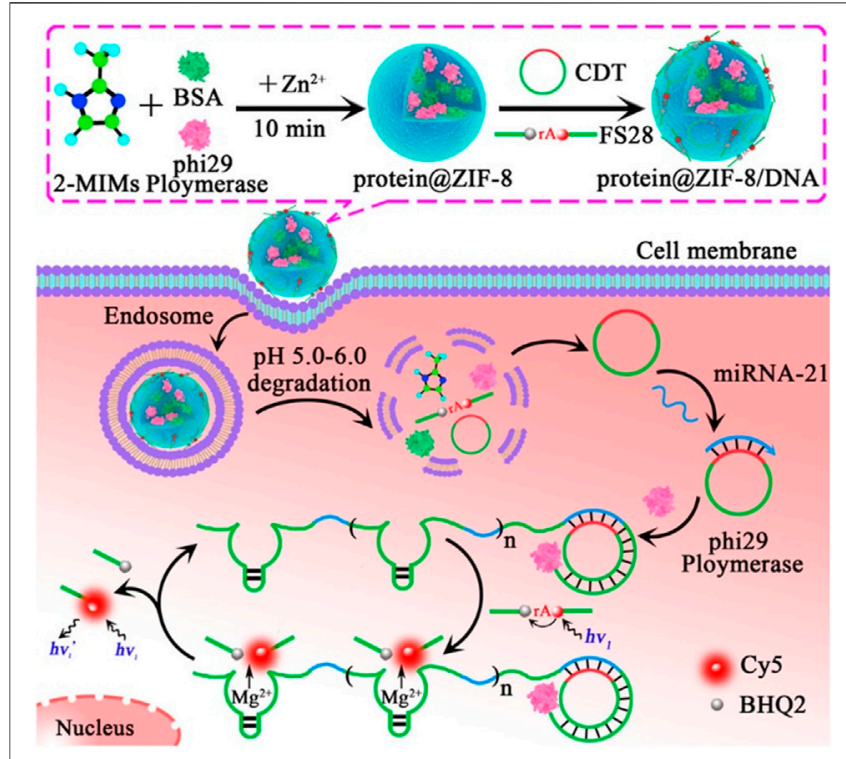

FIGURE 11 | Illustration of fluorescence imaging for microRNA-21 by using ZIF-8 NPs as a nanocarrier for codelivery of $\$ 29$ DNA polymerase and DNA probes in living cells. Reproduced with permission. Copyright 2019, Analytical Chemistry.

release of DOX and augmented the chemotherapy. Meanwhile, the chelation of Mn ions onto PDA shells made this system possible for MRI/PAI, which was proved by both in vitro and in vivo results (Guo et al., 2020).

\section{SENSORS}

Monitoring abnormal changes of molecules during the pathologic process of diseases, such as biomacromolecules, as disease markers, is of great importance for understanding the severity and pathogenesis of diseases. In this chapter, we emphasized the applications of MOFs in biosensors from the following aspects: $\mathrm{FL}, \mathrm{CL}$, and colorimetric methods.

\subsection{Fluorescence Detection}

As carriers, MOFs could deliver FL dye-labeled molecules such as DNA or RNAs to the reaction system. After cargoes in MOFs were reacted with other substances, FL signals were detected. For example, as carriers, ZIF-8 delivered $\phi 29$ DNA polymerase ( $\phi 29 \mathrm{DP})$ and nucleic acid probes to cells, in which $\phi 29 \mathrm{DP}$ was encapsulated into ZIF-8, and nucleic acids were absorbed onto ZIF-8. $\$ 29 \mathrm{DP}$ and nucleic acid probes were released in acidic cellular conditions. In the presence of $\$ 29 \mathrm{DP}$ and deoxyribonucleotide triphosphates (dNTPs), the intracellular miRNA-21 triggered a rolling circle amplification (RCA) reaction and produced an autonomous resultant $\mathrm{Mg}^{2+}$. dependent DNAzyme, which cleaved the fluorogenic substrate and provided a readout FL signal for the monitoring of miRNA21 (Zhang et al., 2019c) (Figure 11).

Except for carriers, MOFs could also be designed as a "gatekeeper" for switch-on/off of fluorophore in FL detection. Wu et al. designed a multicolor detection system with signal dyes loaded and DNA hairpin structure as a capping shell. In this system, as $3^{\prime}$-end of single-strand hairpin-DNA was complementary to DNA probes, FL signals of DNA probes were switched off by the stem-loop structure of hairpin DNA. However, once DNA targets were introduced, FL dyes would be released through a competitive reaction. DNA targets could be detected by monitoring the FL signals with a low detection limit of $20 \mathrm{fM}$. Meanwhile, by loading three different FL dyes with corresponding hairpin DNA, this system enabled multiple DNA detection simultaneously (Wu et al., 2018) (Figure 12). This "switch-on" FL detection is also applicable for other biomolecules. In the platform of AgNPs@PCN-224, AgNPs as a "quencher" reduced the FL of PCN-224; once contacted with $\mathrm{H}_{2} \mathrm{O}_{2}$, AgNPs were etched into silver ions and restored the FL of PCN-224 (Du et al., 2020). Similarly, “switch-off” FL detection achieved by fluorescent MOF such as IRMOF-3 without any modification was also feasible. Azoreductase-responsive AMOF@ MBs with TAMRA (carboxytetramethylrhodamine, donor) and Cy5 (acceptor) closed to each other by the stem-loop structure of $\mathrm{MB}$ was constructed for monitoring VEGF mRNA. In the presence of VEGF mRNA, hybridization of mRNA and stemloop structure disrupted the FL energy resonance transfer (FRET effect), and FL was quenched (Liu et al., 2019a), by which mRNA detection was realized.

In addition, by intrinsic peroxidase-like activity, MOFs could be used for glucose detection combination with FL production. The fluorescent biosensor, ultrathin two-dimensional $\mathrm{Cu}(\mathrm{bpy})_{2}(\mathrm{OTf})_{2}$ nanosheets catalyzed non-fluorescent substrate thiamine $(\mathrm{TH})$ to strong fluorescent thiochrome in the presence of $\mathrm{H}_{2} \mathrm{O}_{2}$ and thus were used for $\mathrm{H}_{2} \mathrm{O}_{2}$ analysis (Shi et al., 2019).

\subsection{Chemiluminescence Detection}

$\mathrm{CL}$ detection is based on light generated during chemical reactions. Luminol, $N$-(4-aminobutyl)- $N$-ethylisoluminol (ABEI), is the most commonly studied substrate for CL. Once oxidized in the presence of $\mathrm{H}_{2} \mathrm{O}_{2}$, it would show strong CL emission. Cu-MOF (Yang et al., 2021a) and Fe-MOF (Dang et al., 2020) (Figure 13) with intrinsic peroxidase activity have been demonstrated persistent $\mathrm{CL}$ through catalyzing the $\mathrm{H}_{2} \mathrm{O}_{2}$-luminol system. Additionally, $\beta$-CD-functionalized MOF (MOF-235/ $\beta$-CD) with excellent catalytic performance exhibited more than 30-fold enhancement in CL intensity. Combined with GOx, this hybrid has been successfully applied for glucose detection (Mao et al., 2018b). Another ultrasensitive CL platform G4/MOFzyme, based on hemin-bridged MOFs, displayed about 100-fold higher catalytic activity to the $\mathrm{H}_{2} \mathrm{O}_{2}$-luminol system. When used in detecting acute myocardial infarction (AMI)-related miRNAs of human serum, their detection limit was lower than $1 \mathrm{fM}$. Moreover, when combined with a smartphone, point-of-care (POC) diagnosis of AMI would be realized, achieving higher sensitivity and longer duration for CL miRNA imaging (Mi et al., 2020).

\subsection{Colorimetric Detection}

Colorimetric detection based on MOFs is mainly dependent on catalytic MOFs. Catalytic MOFs catalyze their substrates such as 


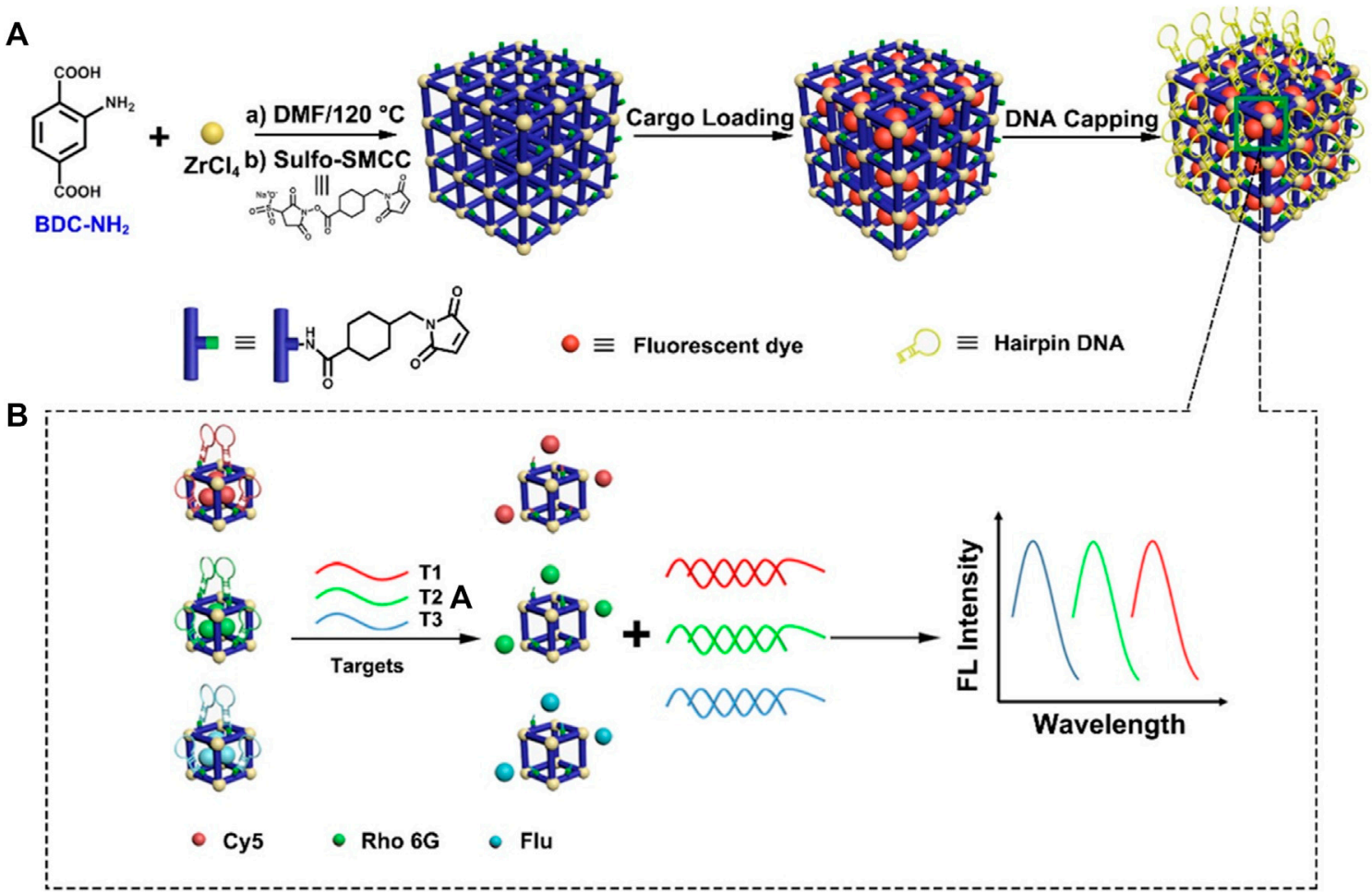

FIGURE 12 | The synthesis and application of metal-organic framework (MOF)-based nanoprobes in DNA multicolor detection. (A) Schematic diagram of synthesis of MOF-based nanoprobes. (B) Diagram of MOF-based probes in multicolor detection of DNA targets. Reproduced with permission. Copyright 2018, Analytical Chemistry.
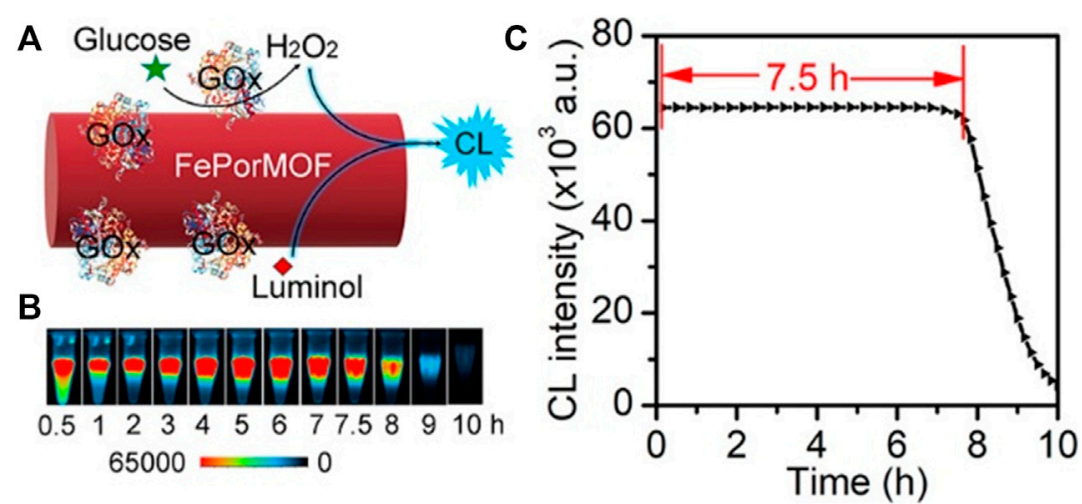

FIGURE 13 | Chemiluminescence detection of nano-/bioenzymes based on FePorMOF/GOx nanoparticles. (A) Principle of chemiluminescence detection based on FePorMOF/GOx. (B) Chemiluminescence (CL) emission of nano-/bioenzymes. (C) Image analysis of FePorMOF/GOx with luminol and glucose. Reproduced with permission. Copyright 2020, Analytical Chemistry.

$3,3^{\prime}, 5,5^{\prime}$-tetramethylbenzidine (TMB) in the presence of $\mathrm{H}_{2} \mathrm{O}_{2}$ to produce an intensive color reaction, which could be quantified through the colorimetric method. These catalytic MOFs could be obtained in three ways: peroxidase delivery (Zheng et al., 2020), nanozyme based on MOFs (Dong et al., 2017a; Chen et al., 2018a), and a combination of both (Zhao et al., 2019a; Xu et al., 2019b; Zhao et al., 2019b).
Wang et al. constructed a $[\mathrm{Cu}(\mathrm{PDA})(\mathrm{DMF})]$ MOF with peroxidase-like activity, which could catalyze colorless substrate TMB to a blue product. In contrast, this phenomenon could be inhibited by dopamine, which could be used for detecting dopamine in human urine and pharmaceutical samples through colorimetric methods (Wang et al., 2019b) (Figure 14). Besides, $\mathrm{PCN}-333(\mathrm{Fe})$, as a carrier of peroxidase 


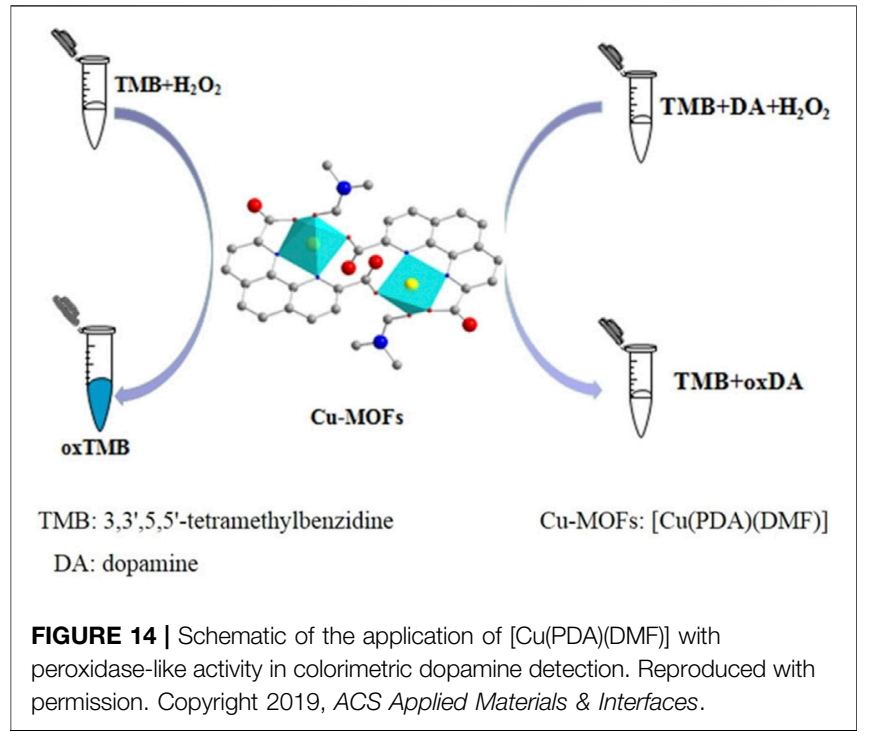

(ficin), showed enhanced peroxidase activity towards TMB oxidation. When combining $\mathrm{H}_{2} \mathrm{O}_{2}$ generated from glucose oxidation, glucose detection was accomplished through a colorimetric method (Zheng et al., 2020).

\section{METAL-ORGANIC FRAMEWORK DERIVATIVES}

In recent years, we also witnessed increasing applications of MOF derivatives in therapeutics and sensors. MOFs mainly exert roles such as sacrificial templates, or forming carbon nanostructures containing porphyrin-like metal centers (PMCS) after hightemperature calcination, or forming ultrasmall nanodots.

MOF derivatives based on MOF as sacrificial templates are for high loading anticancer drugs (Tang et al., 2017). Carbon nanostructure-containing PMCS from MOFs have excellent properties of sonosensitizer for SDT (Pan et al., 2018), photothermal conversion for PTT (Weng et al., 2020) and for CDT/PDT/PTT (Sui et al., 2021), and PAI-guided photothermal/ photodynamic combination therapy (Yang et al., 2018a). Besides, ultrasmall porphyrinic MOF nanodots (MOF QDs) prepared from NMOFs could generate twofold effective ROS and thus benefit enhanced PDT. Interestingly, this ultrasmall nanostructure could be rapidly cleared from the kidney (Wang et al., 2019a).

Furthermore, with abundant atomically dispersed active metal ions and higher photothermal conversion ability, derivatives through pyrolysis have attracted increasing attention in the antibacterial application. For example, a single-atom nanozyme, formed after mesoporous silica $\left(\mathrm{mSiO}_{2}\right)$-protected pyrolysis of ZIF-8, with atomically dispersed active zinc atoms structure, has demonstrated peroxidase-like activity for antibacterial application (Xu et al., 2019a). Ag-doped MOF derivatives (C-Zn/Ag) exhibited a graphitic-like carbon framework after carbonization. Robust photothermal conversion abilities of graphitic-like structure and functions of
$\mathrm{Zn}^{2+}$ and $\mathrm{Ag}^{+}$ions made it possible for dual-functional sterilization (Yang et al., 2020).

\section{CONCLUSION, CHALLENGES, AND PROSPECTS}

In this review, we conclude the most recent applications of MOFs and their composites in nanomedicine. First, various functionalities about MOFs are highlighted, including targeting modification, membrane biomimetics, responsiveness introduction, and other functionalities, aiming at constructing multifunctional MOFs for precise theranostics of diseases. Strategies of MOFs in the treatment of various diseases such as cancer and inflammatory diseases, imaging, sensors, and theranostics have been included without all applications included. Besides, we present applications of MOF derivatives in nanomedicine.

Although fruitful progress has been made in the treatment of diseases during the past few years, the majority of results are focused on cancer treatment, with fewer therapies on other diseases, including inflammatory diseases, antibacterials, and recent summary in regenerative medicine (Shyngys et al., 2021). Therefore, more intelligent therapies based on MOF for the abovementioned diseases are expected. For example, in the treatment for inflammatory diseases such as IBD, nanozymes based on MOF systems are increasingly being applied, in which MOFs are used as carriers or active components. In this therapy, inflammatory microenvironments such as ROS and immune cells were remodeled, and thus inflammation was alleviated. Gene therapy, a promising therapy modality, has been increasingly reported for its personalized treatment of diseases through specific genes involved. However, gene therapy based on MOFs is mainly focused on gene downregulation via siRNA, or CRISPR/Cas9 system, and there has been less coverage in the disease treatment. In addition, plasmid, another gene form, exhibits the capability of both upregulation and downregulation of specific genes but is rarely used, which may be caused by immune obstacles and targeting. MOFs, with superiority such as high loading and modifiability, have great potential in gene therapy. If challenges about MOF delivery of plasmids could be conquered, more smart nanostructures for personalized treatment would be fabricated.

Of course, there is still a long way to go before their clinical transformation, of which toxicity might be the most central factor. In most current reports, the toxicity of MOFs is evaluated through $H \& E$ staining of the main organs for a short term. Along with other crucial parameters including long-term toxicity, accumulation, and their toxicity of MOF components, the pharmacokinetics of MOFs is less frequently being reported, which also needs to be comprehensively considered. In addition, it also takes a longer time to evaluate their usability due to the large differences between species. Therefore, more efforts are needed in MOF platforms, from therapeutic modality exploitation for different diseases to overall toxicity evaluation before their clinical transformation. 


\section{AUTHOR CONTRIBUTIONS}

YM wrote the draft manuscript. XQ and CL provided revised suggestions for this manuscript. QX and KT supervised the manuscript and provided financial support.

\section{FUNDING}

This work was supported by the National Natural Science Foundation of China (No. 32171392), the Natural

\section{REFERENCES}

Abánades Lázaro, I., Haddad, S., Sacca, S., Orellana-Tavra, C., Fairen-Jimenez, D., and Forgan, R. S. (2017). Selective Surface PEGylation of UiO-66 Nanoparticles for Enhanced Stability, Cell Uptake, and pH-Responsive Drug Delivery. Chem 2 (4), 561-578. doi:10.1016/j.chempr.2017.02.005

Abazari, R., Mahjoub, A. R., Ataei, F., Morsali, A., Carpenter-Warren, C. L., Mehdizadeh, K., et al. (2018). Chitosan Immobilization on Bio-MOF Nanostructures: A Biocompatible pH-Responsive Nanocarrier for Doxorubicin Release on MCF-7 Cell Lines of Human Breast Cancer. Inorg. Chem. 57 (21), 13364-13379. doi:10.1021/acs.inorgchem.8b01955

Abuçafy, M. P., Caetano, B. L., Chiari-Andréo, B. G., Fonseca-Santos, B., do Santos, A. M., Chorilli, M., et al. (2018). Supramolecular Cyclodextrin-Based MetalOrganic Frameworks as Efficient Carrier for Anti-Inflammatory Drugs. Eur. J. Pharm. Biopharm. 127, 112-119. doi:10.1016/j.ejpb.2018.02.009

Ahmed, S. A., Nur Hasan, M., Bagchi, D., Altass, H. M., Morad, M., Althagafi, II, et al. (2020). Nano-MOFs as Targeted Drug Delivery Agents to Combat Antibiotic-Resistant Bacterial Infections. R. Soc. Open Sci. 7 (12), 200959. doi:10.1098/rsos.200959

Alsaiari, S. K., Qutub, S. S., Sun, S., Baslyman, W., Aldehaiman, M., Alyami, M., et al. (2021). Sustained and Targeted Delivery of Checkpoint Inhibitors by Metal-Organic Frameworks for Cancer Immunotherapy. Sci. Adv. 7 (4), 10. doi:10.1126/sciadvabe7174

Alyami, M. Z., Alsaiari, S. K., Li, Y., Qutub, S. S., Aleisa, F. A., Sougrat, R., et al. (2020). Cell-Type-Specific CRISPR/Cas9 Delivery by Biomimetic Metal Organic Frameworks. J. Am. Chem. Soc. 142 (4), 1715-1720. doi:10.1021/ jacs.9b11638

An, J., Hu, Y.-G., Cheng, K., Li, C., Hou, X.-L., Wang, G.-L., et al. (2020a). ROSaugmented and Tumor-Microenvironment Responsive Biodegradable Nanoplatform for Enhancing Chemo-Sonodynamic Therapy. Biomaterials 234, 119761. doi:10.1016/j.biomaterials.2020.119761

An, J., Hu, Y.-G., Li, C., Hou, X.-L., Cheng, K., Zhang, B., et al. (2020b). A pH/ Ultrasound Dual-Response Biomimetic Nanoplatform for Nitric Oxide GasSonodynamic Combined Therapy and Repeated Ultrasound for Relieving Hypoxia. Biomaterials 230, 119636. doi:10.1016/j.biomaterials.2019.119636

Bai, J., Peng, C., Guo, L., and Zhou, M. (2019). Metal-Organic FrameworkIntegrated Enzymes as Bioreactor for Enhanced Therapy against Solid Tumor via a Cascade Catalytic Reaction. ACS Biomater. Sci. Eng. 5 (11), 6207-6215. doi:10.1021/acsbiomaterials.9b01200

Bao, J., Zu, X., Wang, X., Li, J., Fan, D., Shi, Y., et al. (2020). Multifunctional Hf/ Mn-TCPP Metal-Organic Framework Nanoparticles for Triple-Modality Imaging-Guided PTT/RT Synergistic Cancer Therapy. Int. J. Nanomed. Vol. 15, 7687-7702. doi:10.2147/IJN.S267321

Butler, K. S., Pearce, C. J., Nail, E. A., Vincent, G. A., and Sava Gallis, D. F. (2020). Antibody Targeted Metal-Organic Frameworks for Bioimaging Applications. ACS Appl. Mater. Inter. 12 (28), 31217-31224. doi:10.1021/acsami.0c07835

Cabrera-García, A., Checa-Chavarria, E., Rivero-Buceta, E., Moreno, V., Fernández, E., and Botella, P. (2019). Amino Modified Metal-Organic Frameworks as $\mathrm{pH}$-Responsive Nanoplatforms for Safe Delivery of Camptothecin. J. Colloid Interf. Sci. 541, 163-174. doi:10.1016/ j.jcis.2019.01.042

Cai, W., Gao, H., Chu, C., Wang, X., Wang, J., Zhang, P., et al. (2017). Engineering Phototheranostic Nanoscale Metal-Organic Frameworks for Multimodal
Science Basic Research Program of Shaanxi (No. 2020JC-36), and the Health Commission of Zhejiang Province (JBZX201901).

\section{SUPPLEMENTARY MATERIAL}

The Supplementary Material for this article can be found online at: https://www.frontiersin.org/articles/10.3389/fmolb.2021.805228/ full\#supplementary-material

Imaging-Guided Cancer Therapy. ACS Appl. Mater. Inter. 9 (3), 2040-2051 doi:10.1021/acsami.6b11579

Cai, Z., Xin, F., Wei, Z., Wu, M., Lin, X., Du, X., et al. (2020). Photodynamic Therapy Combined with Antihypoxic Signaling and CpG Adjuvant as an In Situ Tumor Vaccine Based on Metal-Organic Framework Nanoparticles to Boost Cancer Immunotherapy. Adv. Healthc. Mater. 9 (1), 1900996. doi:10.1002/adhm.201900996

Carrillo-Carrión, C., Martínez, R., Navarro Poupard, M. F., Pelaz, B., Polo, E., Arenas-Vivo, A., et al. (2019). Aqueous Stable Gold Nanostar/ZIF-8 Nanocomposites for Light-Triggered Release of Active Cargo inside Living Cells. Angew. Chem. Int. Ed. 58 (21), 7078-7082. doi:10.1002/anie.201902817

Chen, D., Yang, D., Dougherty, C. A., Lu, W., Wu, H., He, X., et al. (2017a). In Vivo Targeting and Positron Emission Tomography Imaging of Tumor with Intrinsically Radioactive Metal-Organic Frameworks Nanomaterials. ACS Nano 11 (4), 4315-4327. doi:10.1021/acsnano.7b01530

Chen, Q., Xu, M., Zheng, W., Xu, T., Deng, H., and Liu, J. (2017b). Se/Ru-Decorated Porous Metal-Organic Framework Nanoparticles for the Delivery of Pooled siRNAs to Reversing Multidrug Resistance in Taxol-Resistant Breast Cancer Cells. ACS Appl. Mater. Inter. 9 (8), 6712-6724. doi:10.1021/acsami.6b12792

Chen, R., Zhang, J., Chelora, J., Xiong, Y., Kershaw, S. V., Li, K. F., et al. (2017c). Ruthenium(II) Complex Incorporated UiO-67 Metal-Organic Framework Nanoparticles for Enhanced Two-Photon Fluorescence Imaging and Photodynamic Cancer Therapy. ACS Appl. Mater. Inter. 9 (7), 5699-5708. doi:10.1021/acsami.6b12469

Chen, H., Qiu, Q., Sharif, S., Ying, S., Wang, Y., and Ying, Y. (2018a). SolutionPhase Synthesis of Platinum Nanoparticle-Decorated Metal-Organic Framework Hybrid Nanomaterials as Biomimetic Nanoenzymes for Biosensing Applications. ACS Appl. Mater. Inter. 10 (28), 24108-24115. doi:10.1021/acsami.8b04737

Chen, W.-H., Liao, W.-C., Sohn, Y. S., Fadeev, M., Cecconello, A., Nechushtai, R., et al. (2018b). Stimuli-Responsive Nucleic Acid-Based Polyacrylamide Hydrogel-Coated Metal-Organic Framework Nanoparticles for Controlled Drug Release. Adv. Funct. Mater. 28, 1705137. doi:10.1002/adfm.201705137

Chen, W.-H., Luo, G.-F., Vázquez-González, M., Cazelles, R., Sohn, Y. S., Nechushtai, R., et al. (2018c). Glucose-Responsive Metal-OrganicFramework Nanoparticles Act as "Smart" Sense-And-Treat Carriers. ACS Nano 12 (8), 7538-7545. doi:10.1021/acsnano.8b03417

Chen, W.-H., Yang Sung, S., Fadeev, M., Cecconello, A., Nechushtai, R., and Willner, I. (2018d). Targeted VEGF-Triggered Release of an Anti-Cancer Drug from Aptamer-Functionalized Metal-Organic Framework Nanoparticles. Nanoscale 10 (10), 4650-4657. doi:10.1039/c8nr00193f

Chen, X., Tong, R., Shi, Z., Yang, B., Liu, H., Ding, S., et al. (2018e). MOF Nanoparticles with Encapsulated Autophagy Inhibitor in Controlled Drug Delivery System for Antitumor. ACS Appl. Mater. Inter. 10 (3), 2328-2337. doi:10.1021/acsami.7b16522

Chen, H., Yang, J., Sun, L., Zhang, H., Guo, Y., Qu, J., et al. (2019a). Synergistic Chemotherapy and Photodynamic Therapy of Endophthalmitis Mediated by Zeolitic Imidazolate Framework-Based Drug Delivery Systems. Small 15 (47), 1903880. doi:10.1002/smll.201903880

Chen, Y., Liu, W., Shang, Y., Cao, P., Cui, J., Li, Z., et al. (2019b). Folic AcidNanoscale Gadolinium-Porphyrin Metal-Organic Frameworks: Fluorescence and Magnetic Resonance Dual-Modality Imaging and Photodynamic Therapy in Hepatocellular Carcinoma. Int. J. Nanomed. 14, 57-74. doi:10.2147/ IJN.S177880 
Chen, L.-J., Zhao, X., Liu, Y.-Y., and Yan, X.-P. (2020). Macrophage Membrane Coated Persistent Luminescence Nanoparticle@MOF-Derived Mesoporous Carbon Core-Shell Nanocomposites for Autofluorescence-free ImagingGuided Chemotherapy. J. Mater. Chem. B 8 (35), 8071-8083. doi:10.1039/ d0tb01272f

Chen, D., Suo, M., Guo, J., Tang, W., Jiang, W., Liu, Y., et al. (2021). Development of MOF "Armor-Plated" Phycocyanin and Synergistic Inhibition of Cellular Respiration for Hypoxic Photodynamic Therapy in Patient-Derived Xenograft Models. Adv. Healthc. Mater. 10 (3), 2001577. doi:10.1002/adhm.202001577

Cherkasov, V. R., Mochalova, E. N., Babenyshev, A. V., Rozenberg, J. M., Sokolov, I. L., and Nikitin, M. P. (2020). Antibody-Directed Metal-Organic Framework Nanoparticles for Targeted Drug Delivery. Acta Biomater. 103, 223-236. doi:10.1016/j.actbio.2019.12.012

Cho, H., Cho, Y.-Y., Shim, M. S., Lee, J. Y., Lee, H. S., and Kang, H. C. (2020). Mitochondria-Targeted Drug Delivery in Cancers. Biochim. Biophys. Acta (Bba) - Mol. Basis Dis. 1866 (8), 165808. doi:10.1016/j.bbadis.2020.165808

Chowdhuri, A. R., Singh, T., Ghosh, S. K., and Sahu, S. K. (2016). Carbon Dots Embedded Magnetic Nanoparticles @Chitosan @Metal Organic Framework as a Nanoprobe for $\mathrm{pH}$ Sensitive Targeted Anticancer Drug Delivery. ACS Appl. Mater. Inter. 8 (26), 16573-16583. doi:10.1021/acsami.6b03988

Chowdhuri, A. R., Das, B., Kumar, A., Tripathy, S., Roy, S., and Sahu, S. K. (2017). One-pot Synthesis of Multifunctional Nanoscale Metal-Organic Frameworks as an Effective Antibacterial Agent against Multidrug-ResistantStaphylococcus Aureus. Nanotechnology 28 (9), 095102. doi:10.1088/1361-6528/aa57af

Dang, P., Liu, X., Ju, H., and Wu, J. (2020). Intensive and Persistent Chemiluminescence System Based on Nano-/Bioenzymes with Local Tandem Catalysis and Surface Diffusion. Anal. Chem. 92 (7), 5517-5523. doi:10.1021/acs.analchem.0c00337

Dekrafft, K. E., Boyle, W. S., Burk, L. M., Zhou, O. Z., and Lin, W. (2012). Zr- and Hf-Based Nanoscale Metal-Organic Frameworks as Contrast Agents for Computed Tomography. J. Mater. Chem. 22 (35), 18139-18144. doi:10.1039/C2JM32299D

Deng, X., Liang, S., Cai, X., Huang, S., Cheng, Z., Shi, Y., et al. (2019). Yolk-Shell Structured Au Nanostar@Metal-Organic Framework for Synergistic ChemoPhotothermal Therapy in the Second Near-Infrared Window. Nano Lett. 19 (10), 6772-6780. doi:10.1021/acs.nanolett.9b01716

Derlin, T., Grünwald, V., Steinbach, J., Wester, H.-J., and Ross, T. L. (2018). Molecular Imaging in Oncology Using Positron Emission Tomography. Dtsch Arztebl Int. 115 (11), 175-181. doi:10.3238/arztebl.2018.0175

Dong, W., Yang, L., and Huang, Y. (2017a). Glycine Post-Synthetic Modification of MIL-53(Fe) Metal-Organic Framework with Enhanced and Stable PeroxidaseLike Activity for Sensitive Glucose Biosensing. Talanta 167, 359-366. doi:10.1016/j.talanta.2017.02.039

Dong, X., Yu, Y., Wang, Q., Xi, Y., and Liu, Y. (2017b). Interaction Mechanism and Clustering Among RGD Peptides and Integrins. Mol. Inf. 36 (5-6), 1600069. doi:10.1002/minf.201600069

Dong, K., Wang, Z., Zhang, Y., Ren, J., and Qu, X. (2018). Metal-Organic Framework-Based Nanoplatform for Intracellular Environment-Responsive Endo/Lysosomal Escape and Enhanced Cancer Therapy. ACS Appl. Mater. Inter. 10 (38), 31998-32005. doi:10.1021/acsami.8b11972

Dong, K., Zhang, Y., Zhang, L., Wang, Z., Ren, J., and Qu, X. (2019). Facile Preparation of Metal-Organic Frameworks-Based Hydrophobic Anticancer Drug Delivery Nanoplatform for Targeted and Enhanced Cancer Treatment. Talanta 194, 703-708. doi:10.1016/j.talanta.2018.10.101

Du, P., Niu, Q., Chen, J., Chen, Y., Zhao, J., and Lu, X. (2020). "Switch-On" Fluorescence Detection of Glucose with High Specificity and Sensitivity Based on Silver Nanoparticles Supported on Porphyrin Metal-Organic Frameworks. Anal. Chem. 92 (11), 7980-7986. doi:10.1021/ acs.analchem.0c01651

Esfahanian, M., Ghasemzadeh, M. A., and Razavian, S. M. H. (2019). Synthesis, Identification and Application of the Novel Metal-Organic Framework Fe3O4@PAA@ZIF-8 for the Drug Delivery of Ciprofloxacin and Investigation of Antibacterial Activity. Artif. Cell Nanomed. Biotechnol. 47 (1), 2024-2030. doi:10.1080/21691401.2019.1617729

Fang, R. H., Kroll, A. V., Gao, W., and Zhang, L. (2018). Cell Membrane Coating Nanotechnology. Adv. Mater. 30 (23), 1706759. doi:10.1002/adma.201706759

Feng, J., Xu, Z., Dong, P., Yu, W., Liu, F., Jiang, Q., et al. (2019). Stimuli-Responsive Multifunctional Metal-Organic Framework Nanoparticles for Enhanced
Chemo-Photothermal Therapy. J. Mater. Chem. B 7 (6), 994-1004. doi:10.1039/c8tb02815j

Foltz, W. D., and Jaffray, D. A. (2012). Principles of Magnetic Resonance Imaging. Radiat. Res. 177 (4), 331-348. doi:10.1667/rr2620.1

Fu, Q., Zhu, R., Song, J., Yang, H., and Chen, X. (2019). Photoacoustic Imaging: Contrast Agents and Their Biomedical Applications. Adv. Mater. 31 (6), 1805875. doi:10.1002/adma.201805875

Fu, X., Yang, Z., Deng, T., Chen, J., Wen, Y., Fu, X., et al. (2020). A Natural Polysaccharide Mediated MOF-Based Ce6 Delivery System with Improved Biological Properties for Photodynamic Therapy. J. Mater. Chem. B 8 (7), 1481-1488. doi:10.1039/c9tb02482d

Gao, S., Zheng, P., Li, Z., Feng, X., Yan, W., Chen, S., et al. (2018a). Biomimetic O2Evolving Metal-Organic Framework Nanoplatform for Highly Efficient Photodynamic Therapy against Hypoxic Tumor. Biomaterials 178, 83-94. doi:10.1016/j.biomaterials.2018.06.007

Gao, X., Cui, R., Ji, G., and Liu, Z. (2018b). Size and Surface Controllable MetalOrganic Frameworks (MOFs) for Fluorescence Imaging and Cancer Therapy. Nanoscale 10 (13), 6205-6211. doi:10.1039/C7NR08892B

Gao, Z., Chen, F., Li, Y., Zhang, Y., Cheng, K., An, P., et al. (2019). A Small-Sized and Stable 2D Metal-Organic Framework: a Functional Nanoplatform for Effective Photodynamic Therapy. Dalton Trans. 48 (45), 16861-16868. doi:10.1039/c9dt03706c

Gong, M., Yang, J., Li, Y., and Gu, J. (2020). Glutathione-Responsive Nanoscale MOFs for Effective Intracellular Delivery of the Anticancer Drug 6Mercaptopurine. Chem. Commun. 56 (47), 6448-6451. doi:10.1039/d0cc02872j

Guan, Q., Zhou, L.-L., Li, Y.-A., and Dong, Y.-B. (2018). Diiodo-BodipyEncapsulated Nanoscale Metal-Organic Framework for pH-Driven Selective and Mitochondria Targeted Photodynamic Therapy. Inorg. Chem. 57 (16), 10137-10145. doi:10.1021/acs.inorgchem.8b01316

Guo, H., Xia, Y., Feng, K., Qu, X., Zhang, C., and Wan, F. (2020). Surface Engineering of Metal-Organic Framework as pH-/NIR-Responsive Nanocarrier for Imaging-Guided Chemo-Photothermal Therapy. Int. J. Nanomed. 15, 3235-3250. doi:10.2147/IJN.S239910

Gupta, V., Tyagi, S., and Paul, A. K. (2019). Development of Biocompatible IronCarboxylate Metal Organic Frameworks for pH-Responsive Drug Delivery Application. J. Nanosci. Nanotechnol. 19 (2), 646-654. doi:10.1166/ jnn.2019.15402

Guzman-Villanueva, D., and Weissig, V. (2016). Mitochondria-Targeted Agents: Mitochondriotropics, Mitochondriotoxics, and Mitocans. Handb Exp. Pharmacol. 240, 423-438. doi:10.1007/164_2016_37

He, M., Chen, Y., Tao, C., Tian, Q., An, L., Lin, J., et al. (2019a). Mn-PorphyrinBased Metal-Organic Framework with High Longitudinal Relaxivity for Magnetic Resonance Imaging Guidance and Oxygen Self-Supplementing Photodynamic Therapy. ACS Appl. Mater. Inter. 11 (45), 41946-41956. doi:10.1021/acsami.9b15083

He, Z., Dai, Y., Li, X., Guo, D., Liu, Y., Huang, X., et al. (2019b). Hybrid Nanomedicine Fabricated from Photosensitizer-Terminated Metal-Organic Framework Nanoparticles for Photodynamic Therapy and HypoxiaActivated Cascade Chemotherapy. Small 15 (4), 1804131. doi:10.1002/ smll.201804131

He, L., Huang, G., Liu, H., Sang, C., Liu, X., and Chen, T. (2020). Highly Bioactive Zeolitic Imidazolate Framework-8-Capped Nanotherapeutics for Efficient Reversal of Reperfusion-Induced Injury in Ischemic Stroke. Sci. Adv. 6 (12), eaay9751. doi:10.1126/sciadv.aay9751

Hu, C.-M. J., Fang, R. H., Wang, K.-C., Luk, B. T., Thamphiwatana, S., Dehaini, D., et al. (2015). Nanoparticle Biointerfacing by Platelet Membrane Cloaking. Nature 526 (7571), 118-121. doi:10.1038/nature15373

Hu, W. C., Younis, M. R., Zhou, Y., Wang, C., and Xia, X. H. (2020). In Situ Fabrication of Ultrasmall Gold Nanoparticles/2D MOFs Hybrid as Nanozyme for Antibacterial Therapy. Small 16 (23), 2000553. doi:10.1002/ smll.202000553

Huang, C., Tan, W., Zheng, J., Zhu, C., Huo, J., and Yang, R. (2019). AzoreductaseResponsive Metal-Organic Framework-Based Nanodrug for Enhanced Cancer Therapy via Breaking Hypoxia-Induced Chemoresistance. ACS Appl. Mater. Inter. 11 (29), 25740-25749. doi:10.1021/acsami.9b08115

Hussein, E., Zagho, M., Nasrallah, G., and Elzatahry, A. (2018). Recent Advances in Functional Nanostructures as Cancer Photothermal Therapy. Int. J. Nanomed. Vol. 13, 2897-2906. doi:10.2147/IJN.S161031 
Hynek, J., Jurík, S., Koncošová, M., Zelenka, J., Křížová, I., Ruml, T., et al. (2018). The Nanoscaled Metal-Organic Framework ICR-2 as a Carrier of Porphyrins for Photodynamic Therapy. Beilstein J. Nanotechnol. 9, 2960-2967. doi:10.3762/bjnano.9.275

Javanbakht, S., Hemmati, A., Namazi, H., and Heydari, A. (2020). Carboxymethylcellulose-coated 5-fluorouracil@MOF-5 Nano-Hybrid as a Bio-Nanocomposite Carrier for the Anticancer Oral Delivery. Int. J. Biol. Macromolecules 155, 876-882. doi:10.1016/j.ijbiomac.2019.12.007

Jia, X., Yang, Z., Wang, Y., Chen, Y., Yuan, H., Chen, H., et al. (2018). Hollow Mesoporous Silica@Metal-Organic Framework and Applications for pHResponsive Drug Delivery. ChemMedChem 13 (5), 400-405. doi:10.1002/ cmdc.201800019

Jia, Q., Li, Z., Guo, C., Huang, X., Song, Y., Zhou, N., et al. (2019). A $\gamma$-cyclodextrin-based Metal-Organic Framework Embedded with Graphene Quantum Dots and Modified with PEGMA via SI-ATRP for Anticancer Drug Delivery and Therapy. Nanoscale 11 (43), 20956-20967. doi:10.1039/c9nr06195a

Kan, J.-L., Jiang, Y., Xue, A., Yu, Y.-H., Wang, Q., Zhou, Y., et al. (2018). Surface Decorated Porphyrinic Nanoscale Metal-Organic Framework for Photodynamic Therapy. Inorg. Chem. 57 (9), 5420-5428. doi:10.1021/ acs.inorgchem.8b00384

Kundu, T., Mitra, S., Díaz Díaz, D., and Banerjee, R. (2016). Gadolinium(III)-Based Porous Luminescent Metal-Organic Frameworks for Bimodal Imaging. Chempluschem 81 (8), 728-732. doi:10.1002/cplu.201600233

Lai, X., Liu, H., Zheng, Y., Wang, Z., and Chen, Y. (2019). Drug Loaded Nanoparticles of Metal-Organic Frameworks with High Colloidal Stability for Anticancer Application. J. Biomed. Nanotechnol 15 (8), 1754-1763. doi:10.1166/jbn.2019.2807

Lan, G., Ni, K., Xu, Z., Veroneau, S. S., Song, Y., and Lin, W. (2018). Nanoscale Metal-Organic Framework Overcomes Hypoxia for Photodynamic Therapy Primed Cancer Immunotherapy. J. Am. Chem. Soc. 140 (17), 5670-5673. doi:10.1021/jacs.8b01072

Lee, N., Choi, S. H., and Hyeon, T. (2013). Nano-Sized CT Contrast Agents. Adv. Mater. 25 (19), 2641-2660. doi:10.1002/adma.201300081

Lei, B., Wang, M., Jiang, Z., Qi, W., Su, R., and He, Z. (2018). Constructing RedoxResponsive Metal-Organic Framework Nanocarriers for Anticancer Drug Delivery. ACS Appl. Mater. Inter. 10 (19), 16698-16706. doi:10.1021/ acsami.7b19693

Leng, X., Huang, H., Wang, W., Sai, N., You, L., Yin, X., et al. (2018). ZirconiumPorphyrin PCN-222: pH-Responsive Controlled Anticancer Drug Oridonin. Evid.-Based Complement. Altern. Med. 2018, 1-12. doi:10.1155/2018/ 3249023

Li, S., and Huo, F. (2015). Metal-Organic Framework Composites: from Fundamentals to Applications. Nanoscale 7 (17), 7482-7501. doi:10.1039/ c5nr00518c

Li, S.-Y., Cheng, H., Xie, B.-R., Qiu, W.-X., Zeng, J.-Y., Li, C.-X., et al. (2017). Cancer Cell Membrane Camouflaged Cascade Bioreactor for Cancer Targeted Starvation and Photodynamic Therapy. ACS Nano 11 (7), 7006-7018. doi:10.1021/acsnano.7b02533

Li, Y., Xu, N., Zhou, J., Zhu, W., Li, L., Dong, M., et al. (2018). Facile Synthesis of a Metal-Organic Framework Nanocarrier for NIR Imaging-Guided Photothermal Therapy. Biomater. Sci. 6 (11), 2918-2924. doi:10.1039/ c8bm00830b

Li, M., Yue, X., Wang, Y., Zhang, J., Kan, L., and Jing, Z. (2019). Remodeling the Tumor Microenvironment to Improve Drug Permeation and Antitumor Effects by Co-delivering Quercetin and Doxorubicin. J. Mater. Chem. B. 7 (47), 7619-7626. doi:10.1039/c9tb02131k

Li, T., Qiu, H., Liu, N., Li, J., Bao, Y., and Tong, W. (2020a). Construction of SelfActivated Cascade Metal-Organic Framework/Enzyme Hybrid Nanoreactors as Antibacterial Agents. Colloids Surf. B: Biointerfaces 191, 111001. doi:10.1016/ j.colsurfb.2020.111001

Li, X., Salzano, G., Qiu, J., Menard, M., Berg, K., Theodossiou, T., et al. (2020b). Drug-Loaded Lipid-Coated Hybrid Organic-Inorganic "Stealth" Nanoparticles for Cancer Therapy. Front. Bioeng. Biotechnol. 8, 1027. doi:10.3389/ fbioe.2020.01027

Li, Z., Peng, Y., Pang, X., and Tang, B. (2020c). Potential Therapeutic Effects of Mg/ $\mathrm{HCOOH}$ Metal Organic Framework on Relieving Osteoarthritis. ChemMedChem 15 (1), 13-16. doi:10.1002/cmdc.201900546
Lian, X., Erazo-Oliveras, A., Pellois, J.-P., and Zhou, H.-C. (2017). High Efficiency and Long-Term Intracellular Activity of an Enzymatic Nanofactory Based on Metal-Organic Frameworks. Nat. Commun. 8 (1), 2075. doi:10.1038/s41467017-02103-0

Liang, Z., Yang, Z., Yuan, H., Wang, C., Qi, J., Liu, K., et al. (2018). A Protein@ metal-Organic Framework Nanocomposite for $\mathrm{pH}$-Triggered Anticancer Drug Delivery. Dalton Trans. 47 (30), 10223-10228. doi:10.1039/c8dt01789a

Liang, S., Xiao, X., Bai, L., Liu, B., Yuan, M., Ma, P. a., et al. (2021). Conferring TiBased MOFs with Defects for Enhanced Sonodynamic Cancer Therapy. Adv. Mater. 33 (18), 2100333. doi:10.1002/adma.202100333

Lin, Y., Zhong, Y., Chen, Y., Li, L., Chen, G., Zhang, J., et al. (2020). LigandModified Erythrocyte Membrane-Cloaked Metal-Organic Framework Nanoparticles for Targeted Antitumor Therapy. Mol. Pharm. 17 (9), 3328-3341. doi:10.1021/acs.molpharmaceut.0c00421

Liu, M., Wang, L., Zheng, X., Liu, S., and Xie, Z. (2018). Hypoxia-Triggered Nanoscale Metal-Organic Frameworks for Enhanced Anticancer Activity. ACS Appl. Mater. Inter. 10 (29), 24638-24647. doi:10.1021/acsami.8b07570

Liu, N., Zou, Z., Liu, J., Zhu, C., Zheng, J., and Yang, R. (2019a). A Fluorescent Nanoprobe Based on Azoreductase-Responsive Metal-Organic Frameworks for Imaging VEGF mRNA under Hypoxic Conditions. Analyst 144 (21), 6254-6261. doi:10.1039/c9an01671f

Liu, P., Xie, X., Shi, X., Peng, Y., Ding, J., and Zhou, W. (2019b). Oxygen-SelfSupplying and HIF-1 $\alpha$-Inhibiting Core-Shell Nanosystem for HypoxiaResistant Photodynamic Therapy. ACS Appl. Mater. Inter. 11 (51), 48261-48270. doi:10.1021/acsami.9b18112

Liu, W., Pan, Y., Xiao, W., Xu, H., Liu, D., Ren, F., et al. (2019c). Recent Developments on Zinc(ii) Metal-Organic Framework Nanocarriers for Physiological pH-Responsive Drug Delivery. Med. Chem. Commun. 10 (12), 2038-2051. doi:10.1039/c9md00400a

Liu, W. L., Zou, M. Z., Liu, T., Zeng, J. Y., Li, X., Yu, W. Y., et al. (2019d). Expandable Immunotherapeutic Nanoplatforms Engineered from Cytomembranes of Hybrid Cells Derived from Cancer and Dendritic Cells. Adv. Mater. 31 (18), 1900499. doi:10.1002/adma.201900499

Liu, C., Liu, B., Zhao, J., Di, Z., Chen, D., Gu, Z., et al. (2020). Nd ${ }^{3+}$-Sensitized Upconversion Metal-Organic Frameworks for Mitochondria-Targeted Amplified Photodynamic Therapy. Angew. Chem. Int. Ed. 59 (7), 2634-2638. doi:10.1002/anie.201911508

Liu, F., Sheng, S., Shao, D., Xiao, Y., Zhong, Y., Zhou, J., et al. (2021a). A Cationic Metal-Organic Framework to Scavenge Cell-free DNA for Severe Sepsis Management. Nano Lett. 21 (6), 2461-2469. doi:10.1021/acs.nanolett.0c04759

Liu, X., Zhu, X., Qi, X., Meng, X., and Xu, K. (2021b). Co-Administration of iRGD with Sorafenib-Loaded Iron-Based Metal-Organic Framework as a Targeted Ferroptosis Agent for Liver Cancer Therapy. Int. J. Nanomed. Vol. 16, 1037-1050. doi:10.2147/IJN.S292528

Luo, Z., Jiang, L., Yang, S., Li, Z., Soh, W. M. W., Zheng, L., et al. (2019). LightInduced Redox-Responsive Smart Drug Delivery System by Using SeleniumContaining Polymer@MOF Shell/Core Nanocomposite. Adv. Healthc. Mater. 8 (15), 1900406. doi:10.1002/adhm.201900406

Luo, T., Shakya, S., Mittal, P., Ren, X., Guo, T., Bello, M. G., et al. (2020). CoDelivery of Superfine Nano-Silver and Solubilized Sulfadiazine for Enhanced Antibacterial Functions. Int. J. Pharm. 584, 119407. doi:10.1016/ j.ijpharm.2020.119407

Ma, Y., Li, X., Li, A., Yang, P., Zhang, C., and Tang, B. (2017). $\mathrm{H}_{2} \mathrm{~S}$-Activable MOF Nanoparticle Photosensitizer for Effective Photodynamic Therapy against Cancer with Controllable Singlet-Oxygen Release. Angew. Chem. Int. Ed. 56 (44), 13752-13756. doi:10.1002/anie.201708005

Ma, Y.-C., Zhu, Y.-H., Tang, X.-F., Hang, L.-F., Jiang, W., Li, M., et al. (2019). Au Nanoparticles with Enzyme-Mimicking Activity-Ornamented ZIF-8 for Highly Efficient Photodynamic Therapy. Biomater. Sci. 7 (7), 2740-2748. doi:10.1039/ c9bm00333a

Mao, D., Hu, F., KenryJi, S., Wu, W., Ding, D., et al. (2018a). Metal-OrganicFramework-Assisted In Vivo Bacterial Metabolic Labeling and Precise Antibacterial Therapy. Adv. Mater. 30 (18), 1706831. doi:10.1002/ adma.201706831

Mao, X., Lu, Y., Zhang, X., and Huang, Y. (2018b). $\beta$-Cyclodextrin Functionalization of Metal-Organic Framework MOF-235 with Excellent Chemiluminescence Activity for Sensitive Glucose Biosensing. Talanta 188, 161-167. doi:10.1016/j.talanta.2018.05.077 
Meng, H.-M., Hu, X.-X., Kong, G.-Z., Yang, C., Fu, T., Li, Z.-H., et al. (2018). Aptamer-functionalized Nanoscale Metal-Organic Frameworks for Targeted Photodynamic Therapy. Theranostics 8 (16), 4332-4344. doi:10.7150/ thno. 26768

Meng, Z., Huang, H., Huang, D., Zhang, F., and Mi, P. (2021). Functional MetalOrganic Framework-Based Nanocarriers for Accurate Magnetic Resonance Imaging and Effective Eradication of Breast Tumor and Lung Metastasis. J. Colloid Interf. Sci. 581 (Pt A), 31-43. doi:10.1016/j.jcis.2020.07.072

Mi, L., Sun, Y., Shi, L., and Li, T. (2020). Hemin-Bridged MOF Interface with Double Amplification of G-Quadruplex Payload and DNAzyme Catalysis: Ultrasensitive Lasting Chemiluminescence MicroRNA Imaging. ACS Appl. Mater. Inter. 12 (7), 7879-7887. doi:10.1021/acsami.9b18053

Min, H., Wang, J., Qi, Y., Zhang, Y., Han, X., Xu, Y., et al. (2019). Biomimetic Metal-Organic Framework Nanoparticles for Cooperative Combination of Antiangiogenesis and Photodynamic Therapy for Enhanced Efficacy. Adv. Mater. 31 (15), 1808200. doi:10.1002/adma.201808200

Nance, E. (2019). Careers in Nanomedicine and Drug Delivery. Adv. Drug Deliv. Rev. 144, 180-189. doi:10.1016/j.addr.2019.06.009

Ni, K., Luo, T., Lan, G., Culbert, A., Song, Y., Wu, T., et al. (2020). A Nanoscale Metal-Organic Framework to Mediate Photodynamic Therapy and Deliver CpG Oligodeoxynucleotides to Enhance Antigen Presentation and Cancer Immunotherapy. Angew. Chem. Int. Ed. 59 (3), 1108-1112. doi:10.1002/ anie.201911429

Pan, X., Bai, L., Wang, H., Wu, Q., Wang, H., Liu, S., et al. (2018). Metal-OrganicFramework-Derived Carbon Nanostructure Augmented Sonodynamic Cancer Therapy. Adv. Mater. 30 (23), 1800180. doi:10.1002/adma.201800180

Pirovano, G., Roberts, S., Kossatz, S., and Reiner, T. (2020). Optical Imaging Modalities: Principles and Applications in Preclinical Research and Clinical Settings. J. Nucl. Med. 61 (10), 1419-1427. doi:10.2967/ jnumed.119.238279

Qi, Y., Wang, L., Guo, H., Pan, Y., Xie, Z., Jin, N., et al. (2019). Antigen-enabled Facile Preparation of MOF Nanovaccine to Activate the Complement System for Enhanced Antigen-Mediated Immune Response. Biomater. Sci. 7 (10), 4022-4026. doi:10.1039/c9bm01145e

Qin, Y.-T., Feng, Y.-S., Ma, Y.-J., He, X.-W., Li, W.-Y., and Zhang, Y.-K. (2020). Tumor-Sensitive Biodegradable Nanoparticles of Molecularly Imprinted Polymer-Stabilized Fluorescent Zeolitic Imidazolate Framework-8 for Targeted Imaging and Drug Delivery. ACS Appl. Mater. Inter. 12 (22), 24585-24598. doi:10.1021/acsami.0c05154

Qiu, J., Li, X., Steenkeste, K., Barroca-Aubry, N., Aymes-Chodur, C., Roger, P., et al. (2020). Self-assembled Multifunctional Core-Shell Highly Porous MetalOrganic Framework Nanoparticles. Int. J. Pharm. 581, 119281. doi:10.1016/ j.ijpharm.2020.119281

Rahmati, Z., Abdi, J., Vossoughi, M., and Alemzadeh, I. (2020). Ag-doped Magnetic Metal Organic Framework as a Novel Nanostructured Material for Highly Efficient Antibacterial Activity. Environ. Res. 188, 109555. doi:10.1016/ j.envres.2020.109555

Ren, S.-Z., Zhu, D., Zhu, X.-H., Wang, B., Yang, Y.-S., Sun, W.-X., et al. (2019). Nanoscale Metal-Organic-Frameworks Coated by Biodegradable Organosilica for $\mathrm{pH}$ and Redox Dual Responsive Drug Release and High-Performance Anticancer Therapy. ACS Appl. Mater. Inter. 11 (23), 20678-20688. doi:10.1021/acsami.9b04236

Ren, S.-Z., Wang, B., Zhu, X.-H., Zhu, D., Liu, M., Li, S.-K., et al. (2020). Oxygen Self-Sufficient Core-Shell Metal-Organic Framework-Based Smart Nanoplatform for Enhanced Synergistic Chemotherapy and Photodynamic Therapy. ACS Appl. Mater. Inter. 12 (22), 24662-24674. doi:10.1021/ acsami.0c08534

Roth Stefaniak, K., Epley, C. C., Novak, J. J., McAndrew, M. L., Cornell, H. D., Zhu, J., et al. (2018). Photo-triggered Release of 5-fluorouracil from a MOF Drug Delivery Vehicle. Chem. Commun. 54 (55), 7617-7620. doi:10.1039/c8cc01601a

Sava Gallis, D. F., Butler, K. S., Agola, J. O., Pearce, C. J., and McBride, A. A. (2019). Antibacterial Countermeasures via Metal-Organic Framework-Supported Sustained Therapeutic Release. ACS Appl. Mater. Inter. 11 (8), 7782-7791. doi:10.1021/acsami.8b21698

Shakya, S., He, Y., Ren, X., Guo, T., Maharjan, A., Luo, T., et al. (2019). Ultrafine Silver Nanoparticles Embedded in Cyclodextrin Metal-Organic Frameworks with GRGDS Functionalization to Promote Antibacterial and Wound Healing Application. Small 15 (27), 1901065. doi:10.1002/smll.201901065
Shi, M.-Y., Xu, M., and Gu, Z.-Y. (2019). Copper-based Two-Dimensional MetalOrganic Framework Nanosheets as Horseradish Peroxidase Mimics for Glucose Fluorescence Sensing. Anal. Chim. Acta 1079, 164-170. doi:10.1016/ j.aca.2019.06.042

Shi, Z., Zhang, K., Zada, S., Zhang, C., Meng, X., Yang, Z., et al. (2020). Upconversion Nanoparticle-Induced Multimode Photodynamic Therapy Based on a Metal-Organic Framework/Titanium Dioxide Nanocomposite. ACS Appl. Mater. Inter. 12 (11), 12600-12608. doi:10.1021/acsami.0c01467

Shyngys, M., Ren, J., Liang, X., Miao, J., Blocki, A., and Beyer, S. (2021). MetalOrganic Framework (MOF)-Based Biomaterials for Tissue Engineering and Regenerative Medicine. Front. Bioeng. Biotechnol. 9, 603608. doi:10.3389/ fbioe.2021.603608

Sui, C., Tan, R., Chen, Y., Yin, G., Wang, Z., Xu, W., et al. (2021). MOFs-Derived Fe-N Codoped Carbon Nanoparticles as O2-Evolving Reactor and ROS Generator for CDT/PDT/PTT Synergistic Treatment of Tumors. Bioconjug. Chem. 32 (2), 318-327. doi:10.1021/acs.bioconjchem.0c00694

Sun, Q., Bi, H., Wang, Z., Li, C., Wang, X., Xu, J., et al. (2019). Hyaluronic AcidTargeted and pH-Responsive Drug Delivery System Based on Metal-Organic Frameworks for Efficient Antitumor Therapy. Biomaterials 223, 119473. doi:10.1016/j.biomaterials.2019.119473

Swierczewska, M., Han, H. S., Kim, K., Park, J. H., and Lee, S. (2016). Polysaccharide-based Nanoparticles for Theranostic Nanomedicine. Adv. Drug Deliv. Rev. 99 (Pt A), 70-84. doi:10.1016/j.addr.2015.11.015

Tang, L., Shi, J., Wang, X., Zhang, S., Wu, H., Sun, H., et al. (2017). Coordination Polymer Nanocapsules Prepared Using Metal-Organic Framework Templates for pH-Responsive Drug Delivery. Nanotechnology 28 (27), 275601. doi:10.1088/1361-6528/aa7379

Tian, X.-T., Cao, P.-P., Zhang, H., Li, Y.-H., and Yin, X.-B. (2019). GSH-Activated MRI-Guided Enhanced Photodynamic- and Chemo-Combination Therapy with a $\mathrm{MnO}_{2}$-Coated Porphyrin Metal Organic Framework. Chem. Commun. 55 (44), 6241-6244. doi:10.1039/c9cc01957j

Tiwari, A., Singh, A., Garg, N., and Randhawa, J. K. (2017). Curcumin Encapsulated Zeolitic Imidazolate Frameworks as Stimuli Responsive Drug Delivery System and Their Interaction with Biomimetic Environment. Sci. Rep. 7 (1), 12598. doi:10.1038/s41598-017-12786-6

Wang, W., Wang, L., Li, Z., and Xie, Z. (2016). BODIPY-Containing Nanoscale Metal-Organic Frameworks for Photodynamic Therapy. Chem. Commun. 52 (31), 5402-5405. doi:10.1039/c6cc01048b

Wang, L., Zhu, H., Shi, Y., Ge, Y., Feng, X., Liu, R., et al. (2018a). Novel Catalytic Micromotor of Porous Zeolitic Imidazolate Framework-67 for Precise Drug Delivery. Nanoscale 10 (24), 11384-11391. doi:10.1039/c8nr02493f

Wang, S., McGuirk, C. M., d'Aquino, A., Mason, J. A., and Mirkin, C. A. (2018b). Metal-Organic Framework Nanoparticles. Adv. Mater. 30 (37), 1800202. doi:10.1002/adma.201800202

Wang, H., Yu, D., Fang, J., Cao, C., Liu, Z., Ren, J., et al. (2019a). Renal-Clearable Porphyrinic Metal-Organic Framework Nanodots for Enhanced Photodynamic Therapy. ACS Nano 13 (8), 9206-9217. doi:10.1021/acsnano.9b03531

Wang, J., Hu, Y., Zhou, Q., Hu, L., Fu, W., and Wang, Y. (2019b). Peroxidase-like Activity of Metal-Organic Framework [Cu(PDA)(DMF)] and its Application for Colorimetric Detection of Dopamine. ACS Appl. Mater. Inter. 11 (47), 44466-44473. doi:10.1021/acsami.9b17488

Wang, S., Chen, W., Jiang, C., and Lu, L. (2019c). Nanoscaled Porphyrinic MetalOrganic Framework for Photodynamic/photothermal Therapy of Tumor. Electrophoresis 40 (16-17), 2204-2210. doi:10.1002/elps.201900005

Wang, Y., Wu, W., Liu, J., Manghnani, P. N., Hu, F., Ma, D., et al. (2019d). CancerCell-Activated Photodynamic Therapy Assisted by Cu(II)-Based MetalOrganic Framework. ACS Nano 13 (6), 6879-6890. doi:10.1021/ acsnano.9b01665

Wang, X., Zhou, X., Yang, K., Li, Q., Wan, R., Hu, G., et al. (2021). Peroxidase- and UV-Triggered Oxidase Mimetic Activities of the UiO-66-NH2/chitosan Composite Membrane for Antibacterial Properties. Biomater. Sci. 9, 2647-2657. doi:10.1039/d0bm01960g

Weng, Y., Guan, S., Wang, L., Lu, H., Meng, X., Waterhouse, G. I. N., et al. (2020). Defective Porous Carbon Polyhedra Decorated with Copper Nanoparticles for Enhanced NIR-Driven Photothermal Cancer Therapy. Small 16 (1), 1905184. doi:10.1002/smll.201905184

Wu, S., Li, C., Shi, H., Huang, Y., and Li, G. (2018). Design of Metal-Organic Framework-Based Nanoprobes for Multicolor Detection of DNA Targets with 
Improved Sensitivity. Anal. Chem. 90 (16), 9929-9935. doi:10.1021/ acs.analchem.8b02127

Wu, H., Chen, F., Gu, D., You, C., and Sun, B. (2020). A pH-Activated Autocatalytic Nanoreactor for Self-Boosting Fenton-like Chemodynamic Therapy. Nanoscale 12 (33), 17319-17331. doi:10.1039/d0nr03135f

Wu, H., Gu, D., Xia, S., Chen, F., You, C., and Sun, B. (2021a). One-for-all Intelligent Core-Shell Nanoparticles for Tumor-specific PhotothermalChemodynamic Synergistic Therapy. Biomater. Sci. 9 (3), 1020-1033. doi:10.1039/d0bm01734e

Wu, J., Yu, Y., Cheng, Y., Cheng, C., Zhang, Y., Jiang, B., et al. (2021b). LigandDependent Activity Engineering of Glutathione Peroxidase-Mimicking MIL47(V) Metal-Organic Framework Nanozyme for Therapy. Angew. Chem. Int. Ed. 60 (3), 1227-1234. doi:10.1002/anie.202010714

Xiao, Y.-D., Paudel, R., Liu, J., Ma, C., Zhang, Z.-S., and Zhou, S.-K. (2016). MRI Contrast Agents: Classification and Application (Review). Int. J. Mol. Med. 38 (5), 1319-1326. doi:10.3892/ijmm.2016.2744

Xiao, Y., Xu, M., Lv, N., Cheng, C., Huang, P., Li, J., et al. (2021). Dual StimuliResponsive Metal-Organic Framework-Based Nanosystem for Synergistic Photothermal/pharmacological Antibacterial Therapy. Acta Biomater. 122, 291-305. doi:10.1016/j.actbio.2020.12.045

Xie, R., Yang, P., Peng, S., Cao, Y., Yao, X., Guo, S., et al. (2020). A Phosphorylcholine-Based Zwitterionic Copolymer Coated ZIF-8 Nanodrug with a Long Circulation Time and Charged Conversion for Enhanced Chemotherapy. J. Mater. Chem. B 8 (28), 6128-6138. doi:10.1039/d0tb00193g

Xin, H., Wang, F., Luo, R., and Lei, J. (2021). Parallel Lipid Peroxide Accumulation Strategy Based on Bimetal-Organic Frameworks for Enhanced Ferrotherapy. Chem. Eur. J. 27 (13), 4307-4311. doi:10.1002/chem.202005114

Xiong, F., Qin, Z., Chen, H., Lan, Q., Wang, Z., Lan, N., et al. (2020). pH-responsive and Hyaluronic Acid-Functionalized Metal-Organic Frameworks for Therapy of Osteoarthritis. J. Nanobiotechnol. 18 (1), 139. doi:10.1186/s12951-02000694-3

Xu, D., You, Y., Zeng, F., Wang, Y., Liang, C., Feng, H., et al. (2018). Disassembly of Hydrophobic Photosensitizer by Biodegradable Zeolitic Imidazolate Framework-8 for Photodynamic Cancer Therapy. ACS Appl. Mater. Inter. 10 (18), 15517-15523. doi:10.1021/acsami.8b03831

Xu, B., Wang, H., Wang, W., Gao, L., Li, S., Pan, X., et al. (2019a). A Single-Atom Nanozyme for Wound Disinfection Applications. Angew. Chem. Int. Ed. 58 (15), 4911-4916. doi:10.1002/anie.201813994

Xu, W., Jiao, L., Yan, H., Wu, Y., Chen, L., Gu, W., et al. (2019b). Glucose OxidaseIntegrated Metal-Organic Framework Hybrids as Biomimetic Cascade Nanozymes for Ultrasensitive Glucose Biosensing. ACS Appl. Mater. Inter. 11 (25), 22096-22101. doi:10.1021/acsami.9b03004

Xu, Q., Zhan, G., Zhang, Z., Yong, T., Yang, X., and Gan, L. (2021). Manganese Porphyrin-Based Metal-Organic Framework for Synergistic Sonodynamic Therapy and Ferroptosis in Hypoxic Tumors. Theranostics 11 (4), 1937-1952. doi:10.7150/thno.45511

Yan, L., Chen, X., Wang, Z., Zhang, X., Zhu, X., Zhou, M., et al. (2017). Size Controllable and Surface Tunable Zeolitic Imidazolate Framework-8Poly(acrylic Acid Sodium Salt) Nanocomposites for $\mathrm{pH}$ Responsive Drug Release and Enhanced In Vivo Cancer Treatment. ACS Appl. Mater. Inter. 9 (38), 32990-33000. doi:10.1021/acsami.7b10064

Yang, B., Shen, M., Liu, J., and Ren, F. (2017a). Post-Synthetic Modification Nanoscale Metal-Organic Frameworks for Targeted Drug Delivery in Cancer Cells. Pharm. Res. 34 (11), 2440-2450. doi:10.1007/s11095-0172253-9

Yang, Y., Xia, F., Yang, Y., Gong, B., Xie, A., Shen, Y., et al. (2017b). Litchi-Like Fe3O4@Fe-MOF Capped with HAp Gatekeepers for pH-Triggered Drug Release and Anticancer Effect. J. Mater. Chem. B 5 (43), 8600-8606. doi:10.1039/c7tb01680h

Yang, P., Tian, Y., Men, Y., Guo, R., Peng, H., Jiang, Q., et al. (2018a). MetalOrganic Frameworks-Derived Carbon Nanoparticles for Photoacoustic Imaging-Guided Photothermal/Photodynamic Combined Therapy. ACS Appl. Mater. Inter. 10 (49), 42039-42049. doi:10.1021/acsami.8b15828

Yang, Y., Chen, Q., Wu, J.-P., Kirk, T. B., Xu, J., Liu, Z., et al. (2018b). ReductionResponsive Codelivery System Based on a Metal-Organic Framework for Eliciting Potent Cellular Immune Response. ACS Appl. Mater. Inter. 10 (15), 12463-12473. doi:10.1021/acsami.8b01680
Yang, X., Tang, Q., Jiang, Y., Zhang, M., Wang, M., and Mao, L. (2019). Nanoscale ATP-Responsive Zeolitic Imidazole Framework-90 as a General Platform for Cytosolic Protein Delivery and Genome Editing. J. Am. Chem. Soc. 141 (9), 3782-3786. doi:10.1021/jacs.8b11996

Yang, Y., Wu, X., He, C., Huang, J., Yin, S., Zhou, M., et al. (2020). Metal-Organic Framework/Ag-Based Hybrid Nanoagents for Rapid and Synergistic Bacterial Eradication. ACS Appl. Mater. Inter. 12 (12), 13698-13708. doi:10.1021/ acsami.0c01666

Yang, C. P., He, L., Huang, C. Z., Li, Y. F., and Zhen, S. J. (2021a). Continuous Singlet Oxygen Generation for Persistent Chemiluminescence in Cu-MOFsBased Catalytic System. Talanta 221, 121498. doi:10.1016/ j.talanta.2020.121498

Yang, P., Tao, J., Chen, F., Chen, Y., He, J., Shen, K., et al. (2021b). MultienzymeMimic Ultrafine Alloyed Nanoparticles in Metal Organic Frameworks for Enhanced Chemodynamic Therapy. Small 17 (7), 2005865. doi:10.1002/ smll.202005865

Yazdian-Robati, R., Bayat, P., Oroojalian, F., Zargari, M., Ramezani, M., Taghdisi, S. M., et al. (2020). Therapeutic Applications of AS1411 Aptamer, an Update Review. Int. J. Biol. Macromol. 155, 1420-1431. doi:10.1016/ j.ijbiomac.2019.11.118

Yin, X., Yang, B., Chen, B., He, M., and Hu, B. (2019). Multifunctional Gold Nanocluster Decorated Metal-Organic Framework for Real-Time Monitoring of Targeted Drug Delivery and Quantitative Evaluation of Cellular Therapeutic Response. Anal. Chem. 91 (16), 10596-10603. doi:10.1021/ acs.analchem.9b01721

Yin, Y., Yang, J., Pan, Y., Gao, Y., Huang, L., Luan, X., et al. (2021). Mesopore to Macropore Transformation of Metal-Organic Framework for Drug Delivery in Inflammatory Bowel Disease. Adv. Healthc. Mater. 10 (3), 2000973. doi:10.1002/adhm.202000973

Zhang, F.-M., Dong, H., Zhang, X., Sun, X.-J., Liu, M., Yang, D.-D., et al. (2017a). Postsynthetic Modification of ZIF-90 for Potential Targeted Codelivery of Two Anticancer Drugs. ACS Appl. Mater. Inter. 9 (32), 27332-27337. doi:10.1021/ acsami.7b08451

Zhang, H., Chen, W., Gong, K., and Chen, J. (2017b). Nanoscale Zeolitic Imidazolate Framework-8 as Efficient Vehicles for Enhanced Delivery of CpG Oligodeoxynucleotides. ACS Appl. Mater. Inter. 9 (37), 31519-31525. doi:10.1021/acsami.7b09583

Zhang, H., Jiang, W., Liu, R., Zhang, J., Zhang, D., Li, Z., et al. (2017c). Rational Design of Metal Organic Framework Nanocarrier-Based Codelivery System of Doxorubicin Hydrochloride/Verapamil Hydrochloride for Overcoming Multidrug Resistance with Efficient Targeted Cancer Therapy. ACS Appl. Mater. Inter. 9 (23), 19687-19697. doi:10.1021/acsami.7b05142

Zhang, T., Wang, L., Ma, C., Wang, W., Ding, J., Liu, S., et al. (2017d). BODIPYcontaining Nanoscale Metal-Organic Frameworks as Contrast Agents for Computed Tomography. J. Mater. Chem. B 5 (12), 2330-2336. doi:10.1039/ c7tb00392g

Zhang, H., Tian, X.-T., Shang, Y., Li, Y.-H., and Yin, X.-B. (2018a). Theranostic Mn-Porphyrin Metal-Organic Frameworks for Magnetic Resonance ImagingGuided Nitric Oxide and Photothermal Synergistic Therapy. ACS Appl. Mater. Inter. 10 (34), 28390-28398. doi:10.1021/acsami.8b09680

Zhang, L., Wang, Z., Zhang, Y., Cao, F., Dong, K., Ren, J., et al. (2018b). Erythrocyte Membrane Cloaked Metal-Organic Framework Nanoparticle as Biomimetic Nanoreactor for Starvation-Activated Colon Cancer Therapy. ACS Nano 12 (10), 10201-10211. doi:10.1021/acsnano.8b05200

Zhang, W., Lu, J., Gao, X., Li, P., Zhang, W., Ma, Y., et al. (2018c). Enhanced Photodynamic Therapy by Reduced Levels of Intracellular Glutathione Obtained by Employing a Nano-MOF with CuII as the Active Center. Angew. Chem. Int. Ed. 57 (18), 4891-4896. doi:10.1002/anie.201710800

Zhang, Y., Wang, F., Liu, C., Wang, Z., Kang, L., Huang, Y., et al. (2018d). Nanozyme Decorated Metal-Organic Frameworks for Enhanced Photodynamic Therapy. ACS Nano 12 (1), 651-661. doi:10.1021/acsnano.7b07746

Zhang, Y., Wang, L., Liu, L., Lin, L., Liu, F., Xie, Z., et al. (2018e). Engineering Metal-Organic Frameworks for Photoacoustic Imaging-Guided Chemo-/ Photothermal Combinational Tumor Therapy. ACS Appl. Mater. Inter. 10 (48), 41035-41045. doi:10.1021/acsami.8b13492

Zhang, D., Ye, Z., Wei, L., Luo, H., and Xiao, L. (2019a). Cell Membrane-Coated Porphyrin Metal-Organic Frameworks for Cancer Cell Targeting and $\mathrm{O}_{2}$ - 
Evolving Photodynamic Therapy. ACS Appl. Mater. Inter. 11 (43), 39594-39602. doi:10.1021/acsami.9b14084

Zhang, H., Shang, Y., Li, Y.-H., Sun, S.-K., and Yin, X.-B. (2019b). Smart MetalOrganic Framework-Based Nanoplatforms for Imaging-Guided Precise Chemotherapy. ACS Appl. Mater. Inter. 11 (2), 1886-1895. doi:10.1021/ acsami.8b19048

Zhang, J., He, M., Nie, C., He, M., Pan, Q., Liu, C., et al. (2019c). Biomineralized Metal-Organic Framework Nanoparticles Enable Enzymatic Rolling Circle Amplification in Living Cells for Ultrasensitive MicroRNA Imaging. Anal. Chem. 91 (14), 9049-9057. doi:10.1021/acs.analchem.9b01343

Zhang, X., Liu, L., Huang, L., Zhang, W., Wang, R., Yue, T., et al. (2019d). The Highly Efficient Elimination of Intracellular Bacteria via a Metal Organic Framework (MOF)-based Three-In-One Delivery System. Nanoscale 11 (19), 9468-9477. doi:10.1039/c9nr01284b

Zhang, L., Cheng, Q., Li, C., Zeng, X., and Zhang, X.-Z. (2020). Near Infrared LightTriggered Metal Ion and Photodynamic Therapy Based on AgNPs/Porphyrinic MOFs for Tumors and Pathogens Elimination. Biomaterials 248, 120029. doi:10.1016/j.biomaterials.2020.120029

Zhang, L., Gao, Y., Sun, S., Li, Z., Wu, A., and Zeng, L. (2020). pH-Responsive Metal-Organic Framework Encapsulated Gold Nanoclusters with Modulated Release to Enhance Photodynamic Therapy/Chemotherapy in Breast Cancer. J. Mater. Chem. B 8 (8), 1739-1747. doi:10.1039/c9tb02621e

Zhao, H.-X., Zou, Q., Sun, S.-K., Yu, C., Zhang, X., Li, R.-J., et al. (2016). Theranostic Metal-Organic Framework Core-Shell Composites for Magnetic Resonance Imaging and Drug Delivery. Chem. Sci. 7 (8), 5294-5301. doi:10.1039/c6sc01359g

Zhao, Z., Lin, T., Liu, W., Hou, L., Ye, F., and Zhao, S. (2019a). Colorimetric Detection of Blood Glucose Based on GOx@ZIF-8@Fe-Polydopamine Cascade Reaction. Spectrochim. Acta A: Mol. Biomol. Spectrosc. 219, 240-247. doi:10.1016/j.saa.2019.04.061

Zhao, Z., Pang, J., Liu, W., Lin, T., Ye, F., and Zhao, S. (2019b). A Bifunctional Metal Organic Framework of Type Fe(III)-BTC for cascade (Enzymatic and Enzyme-Mimicking) Colorimetric Determination of Glucose. Microchim. Acta 186 (5), 295. doi:10.1007/s00604-019-3416-7

Zhao, J., Yin, F., Ji, L., Wang, C., Shi, C., Liu, X., et al. (2020a). Development of a Tau-Targeted Drug Delivery System Using a Multifunctional Nanoscale MetalOrganic Framework for Alzheimer's Disease Therapy. ACS Appl. Mater. Inter. 12 (40), 44447-44458. doi:10.1021/acsami.0c11064

Zhao, Y., Wang, J., Cai, X., Ding, P., Lv, H., and Pei, R. (2020b). Metal-Organic Frameworks with Enhanced Photodynamic Therapy: Synthesis, Erythrocyte
Membrane Camouflage, and Aptamer-Targeted Aggregation. ACS Appl. Mater. Inter. 12 (21), 23697-23706. doi:10.1021/acsami.0c04363

Zheng, W., Liu, J., Yi, D., Pan, Y., Long, Y., and Zheng, H. (2020). Ficin Encapsulated in Mesoporous Metal-Organic Frameworks with Enhanced Peroxidase-like Activity and Colorimetric Detection of Glucose. Spectrochim. Acta Part A: Mol. Biomol. Spectrosc. 233, 118195. doi:10.1016/j.saa.2020.118195

Zhou, H., Fu, C., Chen, X., Tan, L., Yu, J., Wu, Q., et al. (2018a). MitochondriaTargeted Zirconium Metal-Organic Frameworks for Enhancing the Efficacy of Microwave thermal Therapy against Tumors. Biomater. Sci. 6 (6), 1535-1545. doi:10.1039/c8bm00142a

Zhou, L.-L., Guan, Q., Li, Y.-A., Zhou, Y., Xin, Y.-B., and Dong, Y.-B. (2018b). OnePot Synthetic Approach toward Porphyrinatozinc and Heavy-Atom Involved Zr-NMOF and its Application in Photodynamic Therapy. Inorg. Chem. 57 (6), 3169-3176. doi:10.1021/acs.inorgchem.7b03204

Zhu, Q.-L., and Xu, Q. (2014). Metal-Organic Framework Composites. Chem. Soc. Rev. 43 (16), 5468-5512. doi:10.1039/c3cs60472a

Zhu, Y., Xin, N., Qiao, Z., Chen, S., Zeng, L., Zhang, Y., et al. (2020). Bioactive MOFs Based Theranostic Agent for Highly Effective Combination of Multimodal Imaging and Chemo-Phototherapy. Adv. Healthc. Mater. 9 (14), 2000205. doi:10.1002/adhm.202000205

Zhuang, J., Gong, H., Zhou, J., Zhang, Q., Gao, W., Fang, R. H., et al. (2020). Targeted Gene Silencing In Vivo by Platelet Membrane-Coated Metal-Organic Framework Nanoparticles. Sci. Adv. 6 (13), 10. doi:10.1126/sciadv.aaz6108

Conflict of Interest: The authors declare that the research was conducted in the absence of any commercial or financial relationships that could be construed as a potential conflict of interest.

Publisher's Note: All claims expressed in this article are solely those of the authors and do not necessarily represent those of their affiliated organizations, or those of the publisher, the editors, and the reviewers. Any product that may be evaluated in this article, or claim that may be made by its manufacturer, is not guaranteed or endorsed by the publisher.

Copyright (C) $2021 \mathrm{Ma}, \mathrm{Qu}, \mathrm{Liu}, \mathrm{Xu}$ and Tu. This is an open-access article distributed under the terms of the Creative Commons Attribution License (CC BY). The use, distribution or reproduction in other forums is permitted, provided the original author(s) and the copyright owner(s) are credited and that the original publication in this journal is cited, in accordance with accepted academic practice. No use, distribution or reproduction is permitted which does not comply with these terms. 